\title{
A Wide-angle Glance Of Phosphoglucomutase Gene Family In Pan-Cancer
}

\author{
Ying-nan Liu \\ The First Hospital of China Medical University: The First Affiliated Hospital of China Medical University \\ Shi-xuan Shen \\ The First Hospital of China Medical University: The First Affiliated Hospital of China Medical University \\ Li-rong Yan \\ The First Hospital of China Medical University: The First Affiliated Hospital of China Medical University \\ Han-xi Ding \\ The First Hospital of China Medical University: The First Affiliated Hospital of China Medical University \\ Ang Wang \\ The First Hospital of China Medical University: The First Affiliated Hospital of China Medical University \\ Yuan Yuan \\ The First Hospital of China Medical University: The First Affiliated Hospital of China Medical University \\ Qian Xu ( $\nabla$ qxu@cmu.edu.cn) \\ First Hospital of China Medical University https://orcid.org/0000-0001-8073-6656
}

\section{Primary research}

Keywords: PGM, Pan-Cancer, expression, mutation, prognosis, immune infiltration

Posted Date: May 21st, 2021

DOI: https://doi.org/10.21203/rs.3.rs-540736/v1

License: (a) (i) This work is licensed under a Creative Commons Attribution 4.0 International License. Read Full License 


\section{Abstract \\ Background}

Phosphoglucomutases (PGMs) are a group of important enzymes in glucose metabolism. The PGM family play roles in a variety of cancers, but we still lack a comprehensive understanding of the relationship between the PGM family and pan-cancer.

\section{Methods}

We extracted data from the Cancer Genome Atlas, Oncomine, Human Protein Atlas, and Cancer Cell Line Encyclopedia databases. The R language was mainly used for analysis.

\section{Results}

The PGM1 and PGM5 genes both show low expression in breast cancer, colon cancer, and hepatocellular carcinoma. The expression of PGMs is related to signal transduction pathways, such as protein secretion, ultraviolet (UV) response, and DNA repair pathways, and the infiltration of immune cells such as naive B cells, activated natural killer cells, and resting mast cells in cancers. PGM5 is associated with a higher survival rate in clear-cell renal carcinoma and cervical cancer and a lower survival rate in squamous-cell lung cancer and hepatocellular carcinoma. The copy number variation of PGMs have a broad impact on their expression. In breast cancer, colon cancer, and other cancers, PGM1 is upregulated when it has a higher copy number. Genetic variations in PGMs have adverse effects on the prognosis of various types of cancers. PGM5 mutations in liver cancer and sarcoma and PGM1 copy number deletion in cholangiocarcinoma are all risk factors for those cancers. The qRT-PCR experiments verified the higher expression of $P G M 2 L 1$ and the lower expression of PGM5 in gastric cancer, and the lower expression of PGM1 and PGM5 in colorectal cancer.

\section{Conclusion}

The expression of PGMs is heterogeneous in cancer tissues and is associated with cancer activation pathways as well as immune cell infiltration. The expression of PGMs can affect the prognosis of a variety of cancers. Mutation or copy number variation of PGMs will affect their expression in cancers, as well as the cancer prognosis. These findings provide a foundation for investigating the role of PGMs in the prediction/diagnosis/prognosis of cancers and provide new clues for finding targets for cancer treatment.

\section{Background}

As we know, tumours from the same organ may have many different molecular features. For example, different subtypes of renal cell carcinoma can be accurately classified using differences in the expression of the same miRNA[1]. On the other hand, tumours from different organs often have many identical molecular features. For example, the human TP53 gene is mutated in a wide variety of cancers, including uterine carcinosarcoma, ovarian serous cystadenocarcinoma, oesophageal cancer, and breast cancer[2]. Therefore, it is important to conduct comprehensive longitudinal exploration of certain specific molecules across a variety of tumour types. Pan-cancer analysis is one of the best ways to discover molecular similarities and differences between different types of tumours in various locations in human body, which can identify the molecular characteristics shared by a variety of human cancers and provide new clues about the screening of relevant cancer therapeutic targets.

Phosphoglucomutases (PGMs) are a group of enzymes important in glucose metabolism that are widely present in the human body. The members of the PGM family include PGM1, PGM2, PGM2L1, PGM3, and PGM5. These five proteins have similar functions[3]. In various types of human cancers, metabolic changes are usually observed[4]. As the main enzymes controlling glycolytic and glycogen synthesis in cancer cells, PGMs might be suitable therapeutic targets for specific inhibition of glucose metabolism in cancer cells[5]. They had been reported that PGM1 can inhibit the progression of hepatic carcinoma by controlling glucose transport[6]. PGM1 is highly expressed in lung cancer tissue and is associated with its poor prognosis[7]. PGM3 is associated with breast cancer. Targeting PGM3 to inhibit the hexosamine biosynthetic pathway can lead to growth arrest and apoptosis in breast cancer[8]. PGM5 can be used as a liver cancer[9] and colorectal cancer[10] diagnostic and prognostic biomarker. However, the relationship between the PGM family and human cancer is still limited. Information on PGM expression, genetic variation, and prognosis in pan-cancer is still unclear.

Our aim, in this study, was to comprehensively elucidate the expression, activation pathways, immune cell infiltration, prognosis, and variation of PGMs in pan-cancer and the influence of genetic variations on PGM expression and cancer prognosis, in which we hope to get a wide-angle glance of PGM Family in Pan-Cancer.

\section{Materials And Methods}

\subsection{Data sources}

\subsubsection{TCGA datasets}


The data used in our study were colllected from the TCGA research network (http://cancergenome.nih.gov/). We analysed all 33 specific cancer types (see Additional file: Table S1). All TCGA data, including transcripts per million, mutations, copy number variations (CNVs), and survival information came from UCSC Xena (https://xenabrowser. net/).

\subsubsection{Oncomine database}

The Oncomine database (https://www.oncomine.org/) is a gene microarray data analysis platform. It was used in this study to verify the findings from TCGA.

\subsubsection{The Human Protein Atlas database}

We collected the protein expression data of the PGM genes from the Human Protein Atlas database (https://www.proteinatlas.org/), which includes 20 types of cancers.

\subsubsection{CCLE database}

We analysed the PGM gene expression, mutations, and CNVs in cell lines covered by the CCLE database (https://portals.broadinstitute.org/ccle). Based on the annotations of the different cell lines, they were divided into different cancer types. In all, there were eight cancer types including 325 cell lines.

\subsection{Pan-cancer analysis of the expression of the PGM gene family}

\subsubsection{Pan-cancer analysis of the differential expression characteristics of PGMs}

We used the software package Deseq2 in R to determine the gene expression changes in PGMs in each type of cancer. In the Oncomine analysis, we set the critical values as $\mathrm{P}<0.05$, fold change $>2$, and gene rank $=$ top $10 \%$. In addition, the CCLE database was examined to identify the differential expression of PGMs in different cancer cell lines[11]. We used the Kruskal-Wallis rank test to identify the expression of PGMs in cell lines of different cancer types.

\subsubsection{Pan-cancer analysis of the signal transduction pathways associated with PGM expression}

To analyse the relationship between PGM expression and cancer marker-related pathways, we used gene set variation analysis, which is a nonparametric, unsupervised analytical method that uses expression dataset samples to estimate the enrichment variation within a gene set. We used the Pearson correlation coefficient between pathway activity and PGM gene expression to identify the PGM genes which were associated with the activation or inhibition of certain pathways. In the results, $|r|>0.3$ and $P<0.05$ were considered to show significant correlations.

\subsubsection{Pan-cancer analysis of the correlation between PGMs expression and immune cell infiltration}

To analyse the correlation between immune cell infiltration and PGM expression in diffenent cancers, we calculated the Spearman correlation coefficient between PGMs expression and the level of infiltrating immune cells based on the data provided by the CIBERSORT platform. In the results, $|\mathrm{r}|>0.3$ and $\mathrm{P}$ $<0.05$ were considered to show significant correlations.

\subsubsection{Pan-cancer analysis of the correlation between PGMs expression and prognosis}

In order to analyze the relationship between the expression of PGMs and the prognosis of patients with different cancers, we divided all the cancer patients into two groups based on the median PGMs expression, and the survival rate between the two groups was compared using the log-rank test. In the results, $\mathrm{P}<0.05$ was considered to be statistically significant.

\subsection{Pan-cancer analysis of PGMs mutations and CNVs}

We downloaded the data about PGMs mutation and copy number variation (CNV) frequencies in different cancer tissues from TCGA datasets, as well as the data about PGMs mutation frequencies in different cell lines from the CCLE database. The mutation frequencies and CNV frequencies of PGMs in different types of cancer tissues and cell lines were defined to be the gene mutation proportion and the combined CNV deletion and amplification proportion, respectively.

\subsection{Analysis of the effect of PGMs mutations and CNVs on PGMs expression and cancer prognosis}

We used the Mann-Whitney U test in R software to analyse the effect of PGMs mutations and CNVs on PGMs expression and cancer prognosis.

\subsection{Tissue specimens collection and experimental verification}

We collected clinical specimens from the First Hospital of China Medical University to verify the mRNA expression levels of PGMs. This study was reviewed and approved by the ethics committee of the First Hospital of China Medical University. In total, 60 pairs of gastric cancer and adjacent tissues as well as 40 pairs of colorectal cancer and adjacent tissues were extracted total RNA and tested by real-time quantitative PCR (qRT-PCR) to detect the relative mRNA levels of PGMs in a $20 \mu \mathrm{L}$ reaction system. Take $\beta$-actin as the internal normalization reference of the genes. The fold change was determined via the method of $2-\Delta \Delta \mathrm{Ct}[\Delta \Delta \mathrm{Ct}=(\Delta \mathrm{Ct}$ of PGMs) $-(\Delta \mathrm{Ct}$ of $\beta$-actin)]. The test results were analyzed using the Wilkson test of the non- 
parametric test in SPSS software, and $P<0.05$ was considered statistically significant. See Additional file: Table S2 for the primer sequences of PGMs and $\beta$-actin.

Besides, the Pearson Chi-square test was performed to evaluate the relationship between PGMs expression status and clinicopathological parameters including gender, age, invasive extent, TNM stage, etc. According to the median mRNA expression level, all patients were divided into low expression group and high expression group. The results were analyzed by SPSS software and $P<0.05$ was considered statistically significant.

\section{Results}

\subsection{PGMs expression profiles of in pan-cancer}

\subsubsection{The mRNA expression profile of PGMs}

Based on the continuous variables analysis, we used the data of 33 human tumours derived from TCGA to perform a general analysis of the differential expression of PGMs across different cancer types. The results indicated that the PGMs expression levels were different between the 33 kinds of tumours (Fig. 1A). PGM1 had low expression in breast cancer, colon adenocarcinoma and hepatocellular carcinoma. The $P G M 2 L 1$ gene was highly expressed in gastric adenocarcinoma as well as lung adenocarcinoma. The PGM5 gene had low expression in hepatocellular carcinoma, cervical cancer, clear-cell renal cell carcinoma, prostate cancer, gastric adenocarcinoma, thyroid cancer, papillary renal cell carcinoma, lung adenocarcinoma, head and neck squamouscell carcinoma, breast cancer, lung squamous-cell carcinoma, colon adenocarcinoma, urothelial bladder carcinoma, rectal adenocarcinoma, and endometrial carcinoma. PGM2 and PGM3 did not have significant differential expression between the 33 kinds of tumour tissues. Because the expression of the PGM5 gene was different between most cancers, we visualized the expression of PGM5 in 16 kinds of cancers and normal tissues (Fig. 1B).

We then performed further analyses with the Oncomine data to validate the TCGA results and predict patients' cancer risk (Fig. 2). Consistent with our results from the TCGA data, the expression of the PGM1 and PGM5 genes in various types of cancers still show a decreasing trend. Despite its increased expression in bladder cancer, the PGM1 gene was downregulated to varying degrees in colon cancer, breast cancer, oesophageal cancer, liver cancer, lung cancer, leukaemia and melanoma. Similarly, the PGM5 gene was slightly upregulated in brain and central nervous system cancer and in sarcoma, but it was downregulated in bladder cancer, breast cancer, colon cancer, oesophageal cancer, prostate cancer, lung cancer, cervical cancer, gastric cancer, head and neck cancer, kidney cancer, and ovarian cancer. The PGM2L1 gene was elevated in colorectal cancer, cervical cancer, oesophageal cancer, gastric cancer, head and neck cancer, kidney cancer, pancreatic cancer, and lung adenocarcinoma. The PGM2L1 gene was reduced in bladder cancer, brain and central nervous system cancer, and lymphoma. The PGM2 gene was highly expressed in brain and central nervous system cancer, colon cancer, gastric cancer, and pancreatic cancer but downregulated in oesophageal cancer, leukaemia, and lymphoma. The PGM3 gene was highly expressed in brain and central nervous system cancer, breast cancer, colon cancer, oesophageal cancer, head and neck cancer, prostate cancer and sarcoma, while it was downregulated in leukaemia, liver cancer, lymphoma, and pancreatic cancer.

\subsubsection{The protein expression profile of PGMs}

The immunohistochemistry data from the Human Protein Atlas database demonstrated that a certain amount of PGM protein expression was detected in most tumour tissues (Fig. 3). The expression of PGM proteins was high in melanoma, ovarian cancer and renal cancer, while the protein expression of PGMs in lymphoma and carcinoid was low. Of all the PGMs, PGM3 protein expression was high, and a certain amount of PGM3 protein expression could be detected in all 20 examined tissues, while PGM2L1 protein expression was low, only a small to medium amount being found in some tumour tissues (lung cancer, gastric cancer, pancreatic cancer, etc.). Figure 4 shows the immunohistochemistry results of PGM3 in the Human Protein Atlas tissues from different types of cancer.

\subsubsection{The cellular expression profile of PGMs}

We analyzed the expression of PGMs at the cellular level using the CCLE database, the results indicated that there was a certain level of PGMs expression in 6 cell lines, including bladder cancer, breast cancer, colorectal cancer, gastric cancer, liver cancer, and lung cancer (Fig. 5). The expression levels of the PGM1, PGM2, and PGM3 genes were all high in these six cancer cell lines. PGM2L 1 expression was high only in colorectal cancer cell lines and low in the other five. PGM5 was low in all six cancer cell lines (Fig. 6), which was consistent with the low expression of the PGM5 gene in the corresponding cancers in the TCGA analysis.

\subsection{Signal transduction pathways associated with PGM expression in pan-cancer}

We analyzed the relationship between PGMs expression and cancer-related pathways to elucidate the molecular mechanisms of PGMs in oncogenic processes. The results showed that there was significant correlation between the expression of PGMs and the activity of various cancer pathways (Table 1). PGM1 was mainly involved in haemoglobin metabolism and the hypoxic pathway. PGM2 was mainly involved in pathways including the mitotic spindle, phosphatidylinositol 3-kinase (PI3K)-AKT-mammalian target of rapamycin (mTOR) signalling, the G2/M checkpoint, protein secretion, the E2F target pathway, androgen response, and mTORC1 signalling. PGM3 mainly participated in androgen response, protein secretion, ultraviolet (UV) response and DNA repair pathways. PGM2L1 mainly participated in the transforming growth factor $\beta$ (TGF $\beta$ ) signalling pathway, UV response, the mitotic spindle, the Hedgehog signalling pathway, epithelial-mesenchymal transition, protein secretion, the apical junction complex, reactive oxygen species generation, fatty acid metabolism, DNA repair, and oxidative phosphorylation. PGM5 was mainly involved in myogenesis, UV response, Hedgehog signalling,

Page $4 / 23$ 
pancreatic $\beta$-cells, the MYC-V1 and MYC-V2 target pathways, the E2F target pathway, mTORC1 signalling, the G2/M checkpoint, and DNA repair. PGM1, PGM2, PGM2L1, and PGM3 were more closely related to carcinogenic pathways than the other PGMs. Detailed information on the correlation coefficients and $P$ value between PGMs and cancer-related pathways can be found in Table 1. We also analyzed the correlation between PGMs in the carcinogenic process (Fig. 7A) and found that there may be a synergistic effect between PGMs, especially PGM3, which has significant correlation with the other three PGMs, such as PGM1-PGM3 ( $r=0.44)$, PGM2-PGM3 ( $r=0.45)$, PGM2L1-PGM3 ( $r=0.46)$. In addition, we counted the number of pathways significantly associated with each gene (Fig. 7B), and the results showed that PGM1, PGM2, PGM2L1, PGM3, and PGM5 were all associated with more oncogenic pathways, while PGM2L1, PGM3, and PGM5 were also associated with a small number of tumor suppressor pathways. 
Table 1

Correlation between PGMs expression and signal transduction pathways.

\begin{tabular}{|c|c|c|c|c|c|c|c|c|c|c|}
\hline \multirow[t]{2}{*}{ Pathway } & \multicolumn{2}{|l|}{ PGM1 } & \multicolumn{2}{|l|}{ PGM2 } & \multicolumn{2}{|c|}{ PGM2L1 } & \multicolumn{2}{|l|}{ PGM3 } & \multicolumn{2}{|l|}{ PGM5 } \\
\hline & $\mathbf{r}$ & $\mathbf{P}$ & $\mathbf{r}$ & $\mathbf{P}$ & $\mathbf{r}$ & $\mathbf{P}$ & $\mathbf{r}$ & $\mathbf{P}$ & $\mathbf{r}$ & $\mathbf{P}$ \\
\hline HALLMARK_ADIPOGENESIS & 0.281 & 0 & -0.172 & 0 & -0.259 & 0 & -0.05 & 0 & 0.188 & 0 \\
\hline HALLMARK_ALLOGRAFT_REJECTION & 0.064 & 0 & 0.196 & 0 & 0.073 & 0 & -0.08 & 0 & -0.078 & 0 \\
\hline HALLMARK_ANDROGEN_RESPONSE & 0.189 & 0 & 0.319 & 0 & 0.278 & 0 & 0.516 & 0 & 0.297 & 0 \\
\hline HALLMARK_ANGIOGENESIS & 0.233 & 0 & 0.115 & 0 & 0.299 & 0 & 0.125 & 0 & 0.236 & 0 \\
\hline HALLMARK_APICAL_JUNCTION & 0.178 & 0 & 0.193 & 0 & 0.32 & 0 & 0.089 & 0 & 0.252 & 0 \\
\hline HALLMARK_APICAL_SURFACE & 0.088 & 0 & 0.252 & 0 & 0.148 & 0 & 0.01 & 0.304 & 0.097 & 0 \\
\hline HALLMARK_APOPTOSIS & 0.15 & 0 & 0.267 & 0 & 0.218 & 0 & 0.032 & 0.001 & 0.038 & 0 \\
\hline HALLMARK_BILE_ACID_METABOLISM & 0.27 & 0 & -0.214 & 0 & -0.181 & 0 & 0.099 & 0 & 0.281 & 0 \\
\hline HALLMARK_CHOLESTEROL_HOMEOSTASIS & 0.125 & 0 & 0.076 & 0 & -0.121 & 0 & 0.092 & 0 & -0.086 & 0 \\
\hline HALLMARK_COAGULATION & 0.287 & 0 & -0.018 & 0.064 & 0.064 & 0 & -0.013 & 0.18 & 0.136 & 0 \\
\hline HALLMARK_COMPLEMENT & 0.233 & 0 & 0.193 & 0 & 0.147 & 0 & 0.005 & 0.588 & 0.015 & 0.125 \\
\hline HALLMARK_DNA_REPAIR & -0.168 & 0 & -0.118 & 0 & -0.407 & 0 & -0.317 & 0 & -0.388 & 0 \\
\hline HALLMARK_E2F_TARGETS & -0.121 & 0 & 0.329 & 0 & -0.019 & 0.059 & -0.05 & 0 & -0.423 & 0 \\
\hline HALLMARK_EPITHELIAL_MESENCHYMAL_TRANSITION & 0.215 & 0 & 0.196 & 0 & 0.328 & 0 & 0.155 & 0 & 0.224 & 0 \\
\hline HALLMARK_ESTROGEN_RESPONSE_EARLY & 0.075 & 0 & 0.228 & 0 & 0.216 & 0 & 0.21 & 0 & 0.141 & 0 \\
\hline HALLMARK_ESTROGEN_RESPONSE_LATE & 0.011 & 0.276 & 0.202 & 0 & 0.097 & 0 & 0.092 & 0 & -0.007 & 0.469 \\
\hline HALLMARK_FATTY_ACID_METABOLISM & 0.199 & 0 & -0.165 & 0 & -0.336 & 0 & -0.061 & 0 & 0.078 & 0 \\
\hline HALLMARK_G2M_CHECKPOINT & -0.128 & 0 & 0.409 & 0 & 0.075 & 0 & 0.036 & 0 & -0.372 & 0 \\
\hline HALLMARK_GLYCOLYSIS & 0.194 & 0 & 0.095 & 0 & -0.036 & 0 & -0.032 & 0.001 & -0.249 & 0 \\
\hline HALLMARK_HEDGEHOG_SIGNALING & 0.157 & 0 & 0.014 & 0.157 & 0.411 & 0 & 0.199 & 0 & 0.382 & 0 \\
\hline HALLMARK_HEME_METABOLISM & 0.307 & 0 & 0.029 & 0.004 & 0.138 & 0 & 0.097 & 0 & 0.221 & 0 \\
\hline HALLMARK_HYPOXIA & 0.306 & 0 & 0.182 & 0 & 0.189 & 0 & 0.033 & 0.001 & 0.046 & 0 \\
\hline HALLMARK_IL2_STAT5_SIGNALING & 0.135 & 0 & 0.197 & 0 & 0.159 & 0 & -0.007 & 0.505 & 0.037 & 0 \\
\hline HALLMARK_IL6_JAK_STAT3_SIGNALING & 0.163 & 0 & 0.227 & 0 & 0.117 & 0 & -0.019 & 0.055 & -0.037 & 0 \\
\hline HALLMARK_INFLAMMATORY_RESPONSE & 0.163 & 0 & 0.27 & 0 & 0.197 & 0 & 0.021 & 0.031 & -0.035 & 0 \\
\hline HALLMARK_INTERFERON_ALPHA_RESPONSE & 0.076 & 0 & 0.168 & 0 & -0.009 & 0.342 & -0.091 & 0 & -0.135 & 0 \\
\hline HALLMARK_INTERFERON_GAMMA_RESPONSE & 0.114 & 0 & 0.218 & 0 & 0.057 & 0 & -0.066 & 0 & -0.104 & 0 \\
\hline HALLMARK_KRAS_SIGNALING_DN & 0.018 & 0.073 & 0.101 & 0 & 0.159 & 0 & 0.063 & 0 & 0.109 & 0 \\
\hline HALLMARK_KRAS_SIGNALING_UP & 0.2 & 0 & 0.151 & 0 & 0.282 & 0 & 0.105 & 0 & 0.2 & 0 \\
\hline HALLMARK_MITOTIC_SPINDLE & 0.025 & 0.01 & 0.47 & 0 & 0.386 & 0 & 0.234 & 0 & -0.081 & 0 \\
\hline HALLMARK_MTORC1_SIGNALING & 0.111 & 0 & 0.314 & 0 & -0.038 & 0 & 0.045 & 0 & -0.36 & 0 \\
\hline HALLMARK_MYC_TARGETS_V1 & -0.14 & 0 & 0.258 & 0 & -0.085 & 0 & -0.019 & 0.054 & -0.354 & 0 \\
\hline HALLMARK_MYC_TARGETS_V2 & -0.158 & 0 & 0.128 & 0 & -0.298 & 0 & -0.163 & 0 & -0.434 & 0 \\
\hline HALLMARK_MYOGENESIS & 0.199 & 0 & -0.089 & 0 & 0.153 & 0 & 0.057 & 0 & 0.474 & 0 \\
\hline HALLMARK_NOTCH_SIGNALING & 0.102 & 0 & 0.074 & 0 & 0.289 & 0 & 0.143 & 0 & 0.248 & 0 \\
\hline HALLMARK_OXIDATIVE_PHOSPHORYLATION & 0.027 & 0.005 & -0.276 & 0 & -0.437 & 0 & -0.272 & 0 & -0.102 & 0 \\
\hline HALLMARK_P53_PATHWAY & 0.066 & 0 & 0.203 & 0 & -0.011 & 0.263 & -0.141 & 0 & -0.104 & 0 \\
\hline HALLMARK_PANCREAS_BETA_CELLS & 0.106 & 0 & -0.246 & 0 & 0.111 & 0 & 0.141 & 0 & 0.347 & 0 \\
\hline HALLMARK_PEROXISOME & 0.134 & 0 & -0.177 & 0 & -0.279 & 0 & 0.001 & 0.957 & 0.072 & 0 \\
\hline
\end{tabular}




\begin{tabular}{|c|c|c|c|c|c|c|c|c|c|c|}
\hline \multirow[t]{2}{*}{ Pathway } & \multicolumn{2}{|l|}{ PGM1 } & \multicolumn{2}{|l|}{ PGM2 } & \multicolumn{2}{|c|}{ PGM2L1 } & \multicolumn{2}{|l|}{ PGM3 } & \multicolumn{2}{|l|}{ PGM5 } \\
\hline & $\mathbf{r}$ & $\mathbf{P}$ & $\mathbf{r}$ & $\mathbf{P}$ & $r$ & $\mathbf{P}$ & $\mathbf{r}$ & $\mathbf{P}$ & $\mathbf{r}$ & $\mathbf{P}$ \\
\hline HALLMARK_PI3K_AKT_MTOR_SIGNALING & 0.067 & 0 & 0.421 & 0 & 0.166 & 0 & 0.114 & 0 & -0.075 & 0 \\
\hline HALLMARK_PROTEIN_SECRETION & 0.277 & 0 & 0.329 & 0 & 0.361 & 0 & 0.506 & 0 & 0.2 & 0 \\
\hline HALLMARK_REACTIVE_OXYGEN_SPECIES_PATHWAY & 0.101 & 0 & -0.15 & 0 & -0.3 & 0 & -0.272 & 0 & -0.16 & 0 \\
\hline HALLMARK_SPERMATOGENESIS & -0.126 & 0 & 0.094 & 0 & 0.085 & 0 & 0.016 & 0.106 & -0.24 & 0 \\
\hline HALLMARK_TGF_BETA_SIGNALING & 0.158 & 0 & 0.261 & 0 & 0.47 & 0 & 0.262 & 0 & 0.278 & 0 \\
\hline HALLMARK_TNFA_SIGNALING_VIA_NFKB & 0.15 & 0 & 0.284 & 0 & 0.19 & 0 & 0.026 & 0.009 & -0.038 & 0 \\
\hline HALLMARK_UNFOLDED_PROTEIN_RESPONSE & -0.008 & 0.433 & 0.274 & 0 & -0.07 & 0 & 0.193 & 0 & -0.266 & 0 \\
\hline HALLMARK_UV_RESPONSE_DN & 0.234 & 0 & 0.252 & 0 & 0.492 & 0 & 0.342 & 0 & 0.441 & 0 \\
\hline HALLMARK_UV_RESPONSE_UP & 0.044 & 0 & 0.035 & 0 & -0.089 & 0 & -0.141 & 0 & -0.187 & 0 \\
\hline HALLMARK_WNT_BETA_CATENIN_SIGNALING & -0.105 & 0 & 0.05 & 0 & 0.173 & 0 & 0.047 & 0 & 0.157 & 0 \\
\hline HALLMARK_XENOBIOTIC_METABOLISM & 0.24 & 0 & -0.145 & 0 & -0.216 & 0 & -0.041 & 0 & 0.079 & 0 \\
\hline
\end{tabular}

\subsection{Correlation between PGMs expression and tumour immune cell infiltration in pan- cancer}

Our study explored the correlation between the PGMs expression and the immune cell infiltration status in pan-cancer. The results showed that the expression of PGMs was significantly associated with immune cell infiltration in different cancers. The most relevant immune cells included naive B cells, activated natural killer cells, M0 macrophages, resting mast cells, and activated dendritic cells. Figure 8 shows the relationship between PGMs and the naive $B$ cells in some cancer types.

\subsection{Correlation between PGMs expression and prognosis in pan-cancer}

We explored the prognostic significance of PGMs expression in different cancers. The results indicated that the expression of different PGMs had different prognostic effects in various types of cancers (Fig. 9). The expression of the PGM1 gene was related to a high survival rate from mesothelioma and cervical cancer, as well as a low survival rate from lung squamous-cell carcinoma, lower-grade brain glioma, uveal melanoma, and pancreatic adenocarcinoma. PGM2 gene expression was correlated with a high survival rate from renal clear cell carcinoma and lower-grade brain glioma, as well as a low survival rate from lung adenocarcinoma. $P G M 2 L 1$ gene expression was correlated with a high survival rate from thyroid cancer, as well as a low survival rate from hepatocellular carcinoma, adrenocortical carcinoma, and mesothelioma. The expression of the PGM3 gene was correlated with a low survival rate from sarcomas and adrenocortical carcinoma, as well as a high survival rate from renal papillary carcinoma. PGM5 gene expression was correlated with a clear cell carcinoma and cervical cancer, as well as a low survival rate from adrenocortical carcinoma, hepatocellular carcinoma, lung squamous-cell carcinoma and lung adenocarcinoma.

\subsection{Genovariation characteristics of PGMs in pan-cancer}

We analysed the PGMs mutation frequency based on the TCGA database (Fig. 10A). According to the results, the general average mutation rate of PGMs ranged from 0 to $10.6 \%$. PGM1 and PGM5 showed relatively higher mutation frequencies. PGM1 gene mutations frequently occurred in endometrial cancer, colon cancer, and cutaneous melanoma. PGM5 gene mutations frequently occurred in endometrial cancer, colon cancer, lung adenocarcinoma, bladder urothelial carcinoma, and cutaneous melanoma. In all cancer tissues, endometrial cancer exhibited most often had mutated $P G M$ genes. In contrast, PGM mutation was rare in several cancers, including thymoma, pheochromocytoma, paraganglioma, chromophobe cell renal carcinoma, and cholangiocarcinoma.

We also analysed the CNV frequency of PGMs in different cancer tissues (Fig. 10B). The results indicated that the degree of CNV of PGMs was low in these cancer cells. Relatively speaking, only the $P G M 5$ gene in oesophageal cancer, lung squamous-cell carcinoma, and sarcoma, as well as the $P G M 2 L 1$ gene in bladder urothelial carcinoma, breast cancer, oesophageal cancer, head and neck cancer, lung squamous-cell carcinoma, cutaneous melanoma, and sarcoma, showed multiple copy number amplifications. Only the PGM1 gene in pheochromocytoma and paraganglioma as well as the $P G M 3$ gene in prostate cancer showed a relatively large decrease in copy number.

In addition, we also used the CCLE database to analyse the mutation frequency of PGM genes in eight different cell lines (Fig. 11). The results showed that mutations in PGMs were most commonly found in colon cancer and lung cancer cell lines. Among them, PGM1 had relatively higher mutation frequencies in gastric cancer and lung cancer cell lines; $P G M 2$ had higher mutation frequencies in breast cancer, bladder cancer, colon cancer, and skin cancer cell lines; PGM2L 1 had higher mutation frequencies in breast cancer, colon cancer, gastric cancer, lung cancer, and pancreatic cancer cell lines; $P G M 3$ had a higher mutation frequency only in gastric cancer; and PGM5 had higher mutation frequencies in bladder cancer, kidney cancer, lung cancer, and skin cancer. 


\subsection{Correlation between PGMs genetic variation with PGMs expression and prognosis in pan-cancer}

We separately analyzed the correlation between PGMs mutation, CNV and PGMs expression to investigate whether mutation of PGMs affects their expression. The results indicated that PGMs mutation did not influence the expression of PGMs in most cancers. PGM1 mutation affected its expression only in endometrial carcinoma, and PGM3 mutation affected its expression in hepatocellular cancer and endometrial carcinoma. Compared to mutations, CNVs of PGMs had more effects on their expression in cancers. For example, in bladder urothelial carcinoma, breast cancer, cervical cancer, colon cancer, oesophageal cancer, head and neck cancer, lung squamous-cell carcinoma, lung adenocarcinoma, ovarian cancer, prostate cancer, sarcoma, gastric cancer, and testicular cancer, PGM1 expression was upregulated with copy number amplification (Table 2). The CNVs of PGM2, PGM2L1, PGM3, and PGM5 also had effects on their expression in cancers (see Additional file: Table S3). 
Table 2

Correlation between PGM1 CNV and PGM1 expression.

\begin{tabular}{|c|c|c|c|c|c|}
\hline Gene & Cancer type & CNV & $\mathbf{n}$ & Expression median value & $P$ value \\
\hline \multirow[t]{39}{*}{ PGM1 } & BLCA & DEL & 18 & 10.498(9.889-11.328) & $<0.001$ \\
\hline & & GAIN & 16 & $12.504(11.672-12.796)$ & \\
\hline & & No Change & 374 & $11.408(10.617-12.144)$ & \\
\hline & BRCA & DEL & 57 & $11.157(10.675-11.718)$ & $<0.001$ \\
\hline & & GAIN & 50 & $12.464(12.084-13.355)$ & \\
\hline & & No Change & 983 & $11.772(11.242-12.224)$ & \\
\hline & CESC & DEL & 5 & 10.001(9.994-10.167) & $<0.001$ \\
\hline & & GAIN & 8 & $12.214(12.109-12.855)$ & \\
\hline & & No Change & 281 & $11.716(11.185-12.254)$ & \\
\hline & COAD & DEL & 18 & $10.38(10.141-10.8)$ & 0.001 \\
\hline & & GAIN & 3 & $9.624(9.198-9.949)$ & \\
\hline & & No Change & 441 & $11.122(10.427-11.664)$ & \\
\hline & ESCA & DEL & 10 & $11.164(10.887-11.621)$ & 0.005 \\
\hline & & GAIN & 7 & $12.305(11.877-12.61)$ & \\
\hline & & No Change & 144 & $11.991(11.556-12.396)$ & \\
\hline & HNSC & DEL & 20 & $11.221(10.984-11.433)$ & 0.009 \\
\hline & & GAIN & 10 & $11.791(11.155-12.129)$ & \\
\hline & & No Change & 466 & $11.716(11.289-12.181)$ & \\
\hline & LUAD & DEL & 31 & $11.642(10.964-11.992)$ & 0.024 \\
\hline & & GAIN & 16 & $11.767(10.465-12.672)$ & \\
\hline & & No Change & 477 & $11.944(11.376-12.49)$ & \\
\hline & LUSC & DEL & 27 & $11.375(10.775-11.811)$ & 0.002 \\
\hline & & GAIN & 18 & $12.172(11.694-12.694)$ & \\
\hline & & No Change & 455 & $11.754(11.154-12.284)$ & \\
\hline & OV & DEL & 43 & $11.342(10.772-12.051)$ & $<0.001$ \\
\hline & & GAIN & 49 & $12.664(11.959-13.184)$ & \\
\hline & & No Change & 285 & $12.055(11.555-12.589)$ & \\
\hline & PRAD & DEL & 29 & $11.514(11.174-11.765)$ & $<0.001$ \\
\hline & & GAIN & 1 & $11.823(11.823-11.823)$ & \\
\hline & & No Change & 466 & $12.03(11.579-12.383)$ & \\
\hline & SARC & DEL & 6 & $11.758(11.691-12.434)$ & $<0.001$ \\
\hline & & GAIN & 26 & $12.895(12.41-13.303)$ & \\
\hline & & No Change & 230 & $12.19(11.697-12.683)$ & \\
\hline & STAD & DEL & 10 & $11.711(11.467-11.758)$ & 0.037 \\
\hline & & GAIN & 8 & $12.289(12.117-12.402)$ & \\
\hline & & No Change & 355 & $11.715(11.247-12.2)$ & \\
\hline & TGCT & DEL & 8 & $10.548(9.255-11.78)$ & 0.014 \\
\hline & & GAIN & 3 & $12.716(12.47-13.321)$ & \\
\hline & & No Change & 145 & $11.863(11.134-12.419)$ & \\
\hline
\end{tabular}


We next analysed the relationship between PGMs variations and cancer prognosis. The results showed that mutations and CNVs of PGMs adversely affected the prognosis of some cancers (Table 3,4). PGM mutations were risk factors for cancer, including PGM2L 1 mutations in lower-grade brain glioma and lung adenocarcinoma, PGM2 mutations in bladder cancer, $P G M 3$ mutations in lower-grade brain glioma, $P G M 5$ mutations in hepatic cancer and sarcoma, copy number reduction of $P G M 1$ in cholangiocarcinoma, copy number amplification of $P G M 2$ in uveal melanoma, copy number reduction of $P G M 2 L 1$ in cervical cancer and mesothelioma, copy number reduction of $P G M 3$ in chromophobe renal cell carcinoma, and copy number reduction of $P G M 5$ in acute myeloid leukaemia. Only PGM3 mutation was a protective factor against endometrial cancer, and the deletion of $P G M 3$ was a protective factor against glioblastoma multiforme.

Table 3

Correlation between PGMs mutation and cancer prognosis.

\begin{tabular}{|lllll|}
\hline Gene & HR & 95\%Cl & Cancer type & Pvalue \\
\hline PGM2L1 & 9.689 & $0.022-4239.308$ & LGG & 0.005 \\
PGM2L1 & 2.874 & $0.654-12.624$ & LUAD & 0.015 \\
PGM2 & 3.496 & $0.791-15.441$ & BLCA & 0.001 \\
PGM3 & 57.989 & $0-165962566.166$ & LGG & $<0.001$ \\
\hline PGM3 & 0.157 & $0.068-0.361$ & UCEC & 0.034 \\
\hline PGM5 & 6.454 & $0.195-213.996$ & LIHC & 0.003 \\
\hline PGM5 & 4.432 & $0.419-46.931$ & SARC & 0.005 \\
\hline
\end{tabular}

Table 4

Correlation between PGMs CNV and cancer prognosis.

\begin{tabular}{|llllll|}
\hline Gene & CNV & HR & $\mathbf{9 5 \% C l}$ & Cancer type & $P$ value \\
\hline PGM1 & Del & 10.647 & $1.107-102.41$ & CHOL & 0.041 \\
PGM2 & Gain & 4.119 & $1.377-12.323$ & UVM & 0.011 \\
PGM2L1 & Del & 1.461 & $1.043-2.047$ & CESC & 0.028 \\
PGM2L1 & Del & 1.918 & $1.055-3.49$ & MESO & 0.033 \\
\hline PGM3 & Del & 0.539 & $0.319-0.909$ & GBM & 0.021 \\
\hline PGM3 & Del & 5.397 & $1.625-17.928$ & KICH & 0.006 \\
\hline PGM5 & Del & 5.559 & $1.31-23.593$ & LAML & 0.02 \\
\hline
\end{tabular}

\subsection{Expression validation of PGMs by qRT-PCR experiment and clinicopathological parameter analysis}

In order to verify our analysis results, we performed qRT-PCR experiments to analyze the mRNA expression of PGMs in gastrointestinal cancer tissues. The results showed that, consistent with our TCGA and Oncomine analysis, the expression of $P G M 2 L 1$ in gastric cancer tissues was higher than that in normal gastric tissues $(P=0.036)$; while the expression of $P G M 5$ in gastric cancer tissues was lower than that in normal gastric tissues $(P=0.042)$; and the expression of $\operatorname{PGM1}(P=0.007)$ and $\operatorname{PGM} 5(P<0.001)$ in colorectal cancer were both lower than in normal colorectal tissues. (Fig. 12).

Besides, we further analyzed the relationship between the clinicopathological parameters of gastrointestinal cancer and the expression of PGM gene family. The results showed that no statistically association was found between PGMs expression and the clinicopathological parameters of gastrointestinal cancer (see Additional file: Table S4 and S5).

\section{Discussion}

As the main enzymes controlling glycolysis and glycogen synthesis in cancer cells, PGMs may play an all-purpose role of specific inhibition of glucose metabolism in cancer cells. So far, we have known that PGM1 is involved in glycogen metabolism, catalysing the mutual conversion between 1-phosphate glucose and 6-phosphate glucose and playing a vital role in regulating glucose homeostasis[12]. PGM2 has phosphopentose mutase activity. It is more than 10 times more effective as a phosphopentose mutase than as a glucose phosphate mutase. PGM2L1 has high glucose 1,6-bisphosphate (G16P) synthase activity but low phosphopentose mutase and glucose phosphate mutase activities $(<5 \%)^{[13]}$. PGM3 is mainly involved in the biosynthesis pathway of hexosamine, catalysing the mutual conversion of N-acetylglucosamine 6-phosphate and N-acetylglucosamine 1-phosphate[14]. PGM5 (also called apiculin) is a molecular chaperone of muscular dystrophy proteins ${ }^{[15]}$. PGM5 lacks enzymatic activity but plays a significant role in the formation, maintenance, and repair of myofibrils[16]. However, previous PGM studies were often limited to a single member in a single tumour or a specific pathway[6, 8-10]. With its multidisciplinary analysis employing transcriptomics, proteomics, and cytomics, this study is the first to give a systematic and comprehensive overview of the expression, genetic variation, and activation pathways of the PGM family and their roles in immune cell infiltration and prognosis in human tumours. The results showed that the PGMs are differentially expressed between various carcinoma and corresponding normal

Page $10 / 23$ 
tissues in multilevel data analysis. The expression of PGMs is associated with various signal transduction pathways and immune cell infiltration status. The expression of PGMs can influence the prognosis of many cancers, and mutation or CNV of PGMs can have an impact on their expression in cancers and the prognosis of the patients.

In this study, multi-tier data from TCGA, Oncomine, the Human Protein Atlas, and CCLE were used to perform multidimensional analysis on the mRNA and protein expression levels of PGMs in 33 tumours. In terms of mRNA expression level, the results of our TCGA data analysis and Oncomine database validation showed that the expression of PGMs differs between cancers. PGM1 and PGM5 are significantly downregulated in a variety of tumours, including breast cancer and colon cancer. In addition, we verified the higher expression of $P G M 2 L 1$ and the lower expression of $P G M 5$ in gastric cancer, and the lower expression of PGM1 and PGM5 in colorectal cancer using the qRT-PCR experiments. At the protein expression level, immunohistochemistry showed that the staining intensity of PGMs mostly showed moderate or low intensity in all 20 examined tumour tissues. PGM3 is the most abundantly expressed PGM, with a certain amount of expression observed in all 20 tissues. At the cellular level, PGM2L 1 expression is low in five cell lines but not the colorectal cancer cell line, PGM5 expression is low in all six cancer cell lines, but the expression of the other PGMs in all six cancer cell lines is high. Existing studies support these results. For example, $P G M 1$ has been downregulated in lung cancer cells and hepatic cancer cells[6, 7, 17]. $P G M 5$ is a downregulated tumour suppressor gene in colorectal cancer, suggesting that $P G M 5$ has the potential to be a new predictor and treatment target for colorectal cancer[10]. Our study is the first to describe the expression profiles of PGMs in a variety of cancers, which provides a new perspective for studying PGMs as biomarkers and therapeutic targets of cancer.

To explore the molecular significance of PGMs in carcinogenicity, we analysed the correlation between the expression of PGMs and cancer signal transduction pathways. The results showed that the expression of PGMs is significantly correlated with the inhibition and activation of multiple carcinogenic pathways, such as protein secretion, DNA repair, UV response, the G2/M checkpoint, and the mitotic spindle. PGM1 is mainly involved in haemoglobin metabolism and the hypoxic pathway. PGM2 is mainly involved in pathways including the mitotic spindle, PI3K-AKT-mTOR signalling, the G2/M checkpoint, and protein secretion. PGM5 is mainly involved in pathways including myogenesis, mTORC1 signalling, the G2/M checkpoint, and DNA repair. The pathways most associated with PGM family expression include protein secretion, UV response, and DNA repair. In addition, PGM1, PGM2, PGM2L1, and PGM3 are more closely related to the activation of oncogenic pathways, while PGM5 has more inhibitory effects on oncogenic pathways. In summary, our study showed that the PGM family are involved in various cancer-related signal transduction pathways and play different roles in the occurrence and progression of cancer.

PGMs has been reported to be involved in immune cell responses[18-20], so we analysed the correlation between the expression level of PGMs and the level of immune cell infiltration in pan-cancer. The results showed that the expression of PGMs is significantly associated with the infiltration of multiple immune cells in different cancers, the most relevant of which are naive B cells, activated natural killer cells, resting mast cells, M0 macrophages, and activated dendritic cells. Up to now, few studies have been conducted on the relationship between immune cell infiltration and the $P G M$ gene family, and only the mutation of $P G M 3$ weakens human immunity and increase serum IgE, causing severe combined immunodeficiency syndrome[21-23]. Our study found that the expression of PGM family is closely associated with the level of immune cell infiltration in various cancers. Therefore, the relationship between the PGM family and immune cell infiltration is worth further exploration.

This study analyses the relationship between PGMs and the prognosis of pan-cancer. The results show that the expression of different PGMs has significantly different prognostic significance in a variety of cancers. For example, the expression of the $P G M 1$ gene is correlated with a high survival rate from mesothelioma and cervical cancer and a low survival rate from lung squamous-cell carcinoma, lower-grade brain glioma, uveal melanoma, and pancreatic cancer, while PGM5 gene expression is associated with a high survival rate from renal clear cell carcinoma and cervical cancer and a low survival rate from lung squamous carcinoma, hepatocellular carcinoma, adrenocortical carcinoma, and lung adenocarcinoma. Our findings as mentioned above suggest that both the positive and negative effects of PGMs on prognosis in different cancers may be modified by different cancer tissues,and the mechanism needs further study.

Glycogen metabolism plays an important part in the survival and growth of cancer cells under glucose-depleted or hypoxic conditions[24, 25]. PGM1 is associated with hypoglycaemia-induced accumulation of glycogen in cancer cells[26] and is an essential factor through which cells maintain growth under sustained glucose depletion[27]. Therefore, the expression of PGM1 is closely associated with the development of cancer. The existing literature validates this idea. For example, PGM1 can inhibit the progression of hepatic cancer by regulating the transport of glucose[6]; PGM1 is closely associated with the development of gliomas[28]; endometrial cancer patients are more sensitive to different levels of PGM1 expression[29]; glucose deprivationinduced AMPK activation can induce PGM1 expression, which is associated with poor prognosis in lung cancer patients[7]. In addition, PGM3 is involved in the induction of prostate cancer cell death and may be a potential molecular target for prostate cancer therapeutic agents[30], and Targeting PGM3 to inhibit the hexosamine biosynthetic pathway can lead to growth arrest and apoptosis in breast cancer[8]. Low expression of hepatic PGM5 is associated with poor prognosis in hepatic cancer patients; PGM5 has the potential to be a prognostic and diagnostic biomarker of hepatic cancer[9]; low expression of PGM5 is also associated with poor prognosis in colorectal cancer patients; and the upregulation of PGM5 inhibits the progression of proliferation, migration, and invasion of colorectal cancer cells, indicating that PGM5 can serve as a potential novel prediction and therapeutic target for colorectal cancer[10]. Our results indicated that,as a group of important enzymes in glucose metabolism, PGMs play significant roles in various cancer types. It suggested that metabolic changes are important hallmarks of cancer cells. The potential of PGMs as prognostic predictors and therapeutic targets in different cancers is worth further investigation.

This study also analysed the mutation and CNV frequencies of PGMs in pan-cancer. The results indicated that the overall average mutation rate of PGMs ranged from 0 to $10.6 \%$, in which the mutation frequencies of $P G M 1$ and $P G M 5$ are relatively higher. The mutation frequency of all PGMs is relatively high 
in endometrial cancer, which is a cancer with a high global mutation burden[31]. In contrast, mutations of PGMs are very rare in thymoma, pheochromocytoma, paraganglioma, chromophobe renal cell carcinoma, and cholangiocarcinoma. The CNV frequencies of PGMs are relatively low. PGM2L 1 exhibits more copy number amplification in cancers such as breast cancer, oesophageal cancer, head and neck cancer, squamous-cell carcinoma, and adenocarcinoma of the cervix, while PGM3 shows a high frequency of copy number reduction in prostate cancer. No CNV in PGMs is observed in renal clear cell carcinoma, papillary renal cell carcinoma, thyroid carcinoma, or thymoma. We also analysed the mutations of PGMs in human cancer cell lines which indicated that PGM mutations are more common in lung cancer and colorectal cancer cell lines. These results revealed the genetic variation characteristics of PGMs across cancer types, suggesting that variations in PGMs may be associated with the genesis and progression of certain cancers.

When we analysed the effect of $P G M$ variations on the expression of PGMs and the pan-cancer prognosis, we found that $P G M$ mutations do not affect the expression of PGMs in most cancers; PGM1 mutation only affects its expression in endometrial carcinoma, and PGM3 mutation only affects its expression in hepatocellular carcinoma and endometrial cancer. The CNVs of PGMs can widely affect their expression in a variety of cancers, including breast cancer, cervical cancer, and colon cancer, and PGM expression is usually upregulated with increasing copy number. The burden of tumour copy number change is an important prognostic factor related to the recurrence and death of pan-cancer[32]. Therefore, we analysed the effects of $P G M$ variations on the prognosis of pan-cancer. The results showed that mutation and CNV of PGMs could affect the prognosis of some cancers, and most of them have adverse effects. Among them, mutation of $P G M 3$ is a protective factor against endometrial cancer. The increase in the copy number of $P G M 2 L 1$ is a risk factor for squamous-cell carcinoma and adenocarcinoma of the cervix. According to the above findings, the mutation frequency of $P G M 3$ in endometrial cancer is high, and the copy number amplification frequency of $P G M 2 L 1$ in squamous-cell carcinoma and adenocarcinoma of the cervix is relatively high. Therefore, we may conclude that mutations in PGM3 and copy number amplification of $P G M 2 L 1$ could serve as prognostic predictors for endometrial cancer and squamous-cell carcinoma and adenocarcinoma of the cervix, respectively.

\section{Conclusions}

Overall, our study provides comprehensive and systematic information on the expression, mutation, CNV, and activation pathways of PGMs and their associations with immune cell infiltration and prognosis in pan-cancer. This study provides detailed analytical data with which to explore the correlation between PGM and metabolic imbalance in human tumours and their role in the specific inhibition of glucose metabolism in cancer cells. Our study expands the foundation on which to explore the feasibility of PGMs as predictive/diagnostic/prognostic factors for cancers and provides new clues for the screening of relevant cancer therapeutic targets.

\section{Abbreviations}

PGM, Phosphoglucomutase; TCGA, the Cancer Genome Atlas; CCLE, Cancer Cell Line Encyclopedia; CNV, copy number variation.

\section{Declarations}

\section{Ethics approval and consent to participate}

All participants involved in this study have provided written informed consent and the study protocols were permitted by the Ethics Committee of the First Hospital of China Medical University and performed in accordance with the Declaration of Helsink

\section{Consent for publication}

Not applicable

\section{Availability of data and materials}

The datasets generated and analysed during the current study are available in the TCGA datasets, [http://cancergenome.nih.gov/]区 Oncomine database, [ https://www.oncomine.org/]; the Human Protein Atlas database, [ https://www.proteinatlas.org/]; and the CCLE database [https://portals.broadinstitute.org/ccle]. Other people could use these data.

\section{Competing interests}

The authors declare that they have no competing interests

\section{Funding}

This work was supported by the National Key R\&D Program of China (Grant 2018YFC1311600) and Liaoning Revitalization Talents Program (Grant \#XLYC1808036).

\section{Authors' contributions}

Quan Xu and Yuan Yuan designed the study and revised the manuscript; Ying-nan Liu collected/analyzed the data and drafted the manuscript; Han-xi Ding, Shi-xuan Shen, Li-rong Yan, and Ang Wang was involved in data analysis. All authors read and approved the final manuscript. 


\section{Acknowledgements}

Not applicable

\section{References}

1. Youssef YM, White NM, Grigull J, Krizova A, Samy C, Mejia-Guerrero S, et al. Accurate molecular classification of kidney cancer subtypes using microRNA signature. Eur Urol. 2011; 59: 721-30.

2. Wang X SQ. TP53 mutations, expression and interaction networks in human cancers. Oncotarget. 2017 Jan; 8(1): 624-43.

3. Muenks AG, Stiers KM, Beamer LJ. Sequence-structure relationships, expression profiles, and disease-associated mutations in the paralogs of phosphoglucomutase 1. PLoS One. 2017; 12: e0183563.

4. Weyandt JD, Thompson CB, Giaccia AJ, Rathmell WK. Metabolic Alterations in Cancer and Their Potential as Therapeutic Targets. Am Soc Clin Oncol Educ Book. 2017; 37: 825-32.

5. Marin-Hernandez A, Gallardo-Perez JC, Reyes-Garcia MA, Sosa-Garrocho M, Macias-Silva M, Rodriguez-Enriquez S, et al. Kinetic modeling of glucose central metabolism in hepatocytes and hepatoma cells. Biochim Biophys Acta Gen Subj. 2020; 1864: 129687.

6. Jin GZ, Zhang Y, Cong WM, Wu X, Wang X, Wu S, et al. Phosphoglucomutase 1 inhibits hepatocellular carcinoma progression by regulating glucose trafficking. PLoS Biol. 2018; 16: e2006483.

7. Li Y, Liang R, Sun M, Li Z, Sheng H, Wang J, et al. AMPK-dependent phosphorylation of HDAC8 triggers PGM1 expression to promote lung cancer cell survival under glucose starvation. Cancer Lett. 2020; 478: 82-92.

8. Ricciardiello F, Votta G, Palorini R, Raccagni I, Brunelli L, Paiotta A, et al. Inhibition of the Hexosamine Biosynthetic Pathway by targeting PGM3 causes breast cancer growth arrest and apoptosis. Cell Death Dis. 2018; 9: 377.

9. Jiao Y, Li Y, Jiang P, Han W, Liu Y. PGM5: a novel diagnostic and prognostic biomarker for liver cancer. PeerJ. 2019 ; 7: e7070.

10. Sun Y, Long H, Sun L, Sun X, Pang L, Chen J, et al. PGM5 is a promising biomarker and may predict the prognosis of colorectal cancer patients. Cancer Cell Int. 2019; 19: 253.

11. Ghandi M, Huang FW, Jane-Valbuena J, Kryukov GV, Lo CC, McDonald ER, 3rd, et al. Next-generation characterization of the Cancer Cell Line Encyclopedia. Nature. 2019; 569: 503-8.

12. Beamer LJ. Mutations in hereditary phosphoglucomutase 1 deficiency map to key regions of enzyme structure and function. J Inherit Metab Dis. 2015; 38: 243-56.

13. Maliekal P, Sokolova T, Vertommen D, Veiga-da-Cunha M, Van Schaftingen E. Molecular identification of mammalian phosphopentomutase and glucose-1,6-bisphosphate synthase, two members of the alpha-D-phosphohexomutase family. J Biol Chem. 2007; 282: 31844-51.

14. Greig KT, Antonchuk J, Metcalf D, Morgan PO, Krebs DL, Zhang JG, et al. Agm1/Pgm3-mediated sugar nucleotide synthesis is essential for hematopoiesis and development. Mol Cell Biol. 2007; 27: 5849-59.

15. A M B. Association of aciculin with dystrophin and utrophin. \%J The Journal of biological chemistry. 1995; 11: 6328-37.

16. Molt S, Buhrdel JB, Yakovlev S, Schein P, Orfanos Z, Kirfel G, et al. Aciculin interacts with filamin C and Xin and is essential for myofibril assembly, remodeling and maintenance. J Cell Sci. 2014; 127: 3578-92.

17. Schroder PC, Segura V, Riezu JI, Sangro B, Mato JM, Prieto J, et al. A signature of six genes highlights defects on cell growth and specific metabolic pathways in murine and human hepatocellular carcinoma. Funct Integr Genomics. 2011; 11: 419-29.

18. Fleisher TA. Autosomal Recessive Phosphoglucomutase 3 (PGM3) Mutations Link Glycosylation Defects to Atopy, Immune Deficiency, Autoimmunity, and Neurocognitive Impairment. Pediatrics. 2014; 134 Suppl 3: S181-2.

19. Yang L, Fliegauf M, Grimbacher B. Hyper-IgE syndromes: reviewing PGM3 deficiency. Curr Opin Pediatr. 2014; 26: 697-703.

20. Zhang Y, Yu X, Ichikawa M, Lyons JJ, Datta S, Lamborn IT, et al. Autosomal recessive phosphoglucomutase 3 (PGM3) mutations link glycosylation defects to atopy, immune deficiency, autoimmunity, and neurocognitive impairment. J Allergy Clin Immunol. 2014; 133: 1400-9, 9 e1-5.

21. Sassi A, Lazaroski S, Wu G, Haslam SM, Fliegauf M, Mellouli F, et al. Hypomorphic homozygous mutations in phosphoglucomutase 3 (PGM3) impair immunity and increase serum IgE levels. J Allergy Clin Immunol. 2014; 133: 1410-9, 9 e1-13.

22. Smart BA. Deficiency of Innate and Acquired Immunity Caused by an IKBKB Mutation. Pediatrics. 2014; 134 Suppl 3: S181.

23. Pacheco-Cuellar G, Gauthier J, Desilets V, Lachance C, Lemire-Girard M, Rypens F, et al. A Novel PGM3 Mutation Is Associated With a Severe Phenotype of Bone Marrow Failure, Severe Combined Immunodeficiency, Skeletal Dysplasia, and Congenital Malformations. J Bone Miner Res. 2017; 32: 1853-9.

24. Shen GM, Zhang FL, Liu XL, Zhang JW. Hypoxia-inducible factor 1-mediated regulation of PPP1R3C promotes glycogen accumulation in human MCF-7 cells under hypoxia. FEBS Lett. 2010; 584: 4366-72.

25. Favaro E, Bensaad K, Chong MG, Tennant DA, Ferguson DJ, Snell C, et al. Glucose utilization via glycogen phosphorylase sustains proliferation and prevents premature senescence in cancer cells. Cell Metab. 2012; 16: 751-64.

26. Pelletier J, Bellot G, Gounon P, Lacas-Gervais S, Pouyssegur J, Mazure NM. Glycogen Synthesis is Induced in Hypoxia by the Hypoxia-Inducible Factor and Promotes Cancer Cell Survival. Front Oncol. 2012; 2: 18. 
27. Bae E, Kim HE, Koh E, Kim KS. Phosphoglucomutase1 is necessary for sustained cell growth under repetitive glucose depletion. FEBS Lett. 2014; 588: 3074-80.

28. Kounelakis MG, Zervakis ME, Giakos GC, Postma GJ, Buydens LM, Kotsiakis X. On the relevance of glycolysis process on brain gliomas. IEEE J Biomed Health Inform. 2013; 17: 128-35.

29. Marshall M, Neal F, Goldberg D. Isoenzymes of hexokinase, 6-phosphogluconate dehydrogenase, phosphoglucomutase and lactate dehydrogenase in uterine cancer. Br J Cancer. 1979; 40(3): 380-90.

30. Lee C, Jeong SJ, Yun SM, Kim JH, Lee HJ, Ahn KS, et al. Down-regulation of phosphoglucomutase 3 mediates sulforaphane-induced cell death in LNCaP prostate cancer cells. . Proteome Sci. 2010: 8-67.

31. Rutgers J. Update on pathology, staging and molecular pathol-ogy of endometrial (uterine corpus) adenocarcinoma. Future Oncol. 2015; 11(23): 3207-18.

32. Hieronymus H, Murali R, Tin A, Yadav K, Abida W, Moller H, et al. Tumor copy number alteration burden is a pan-cancer prognostic factor associated with recurrence and death. Elife. 2018; 7: e37294.

\section{Figures}

A

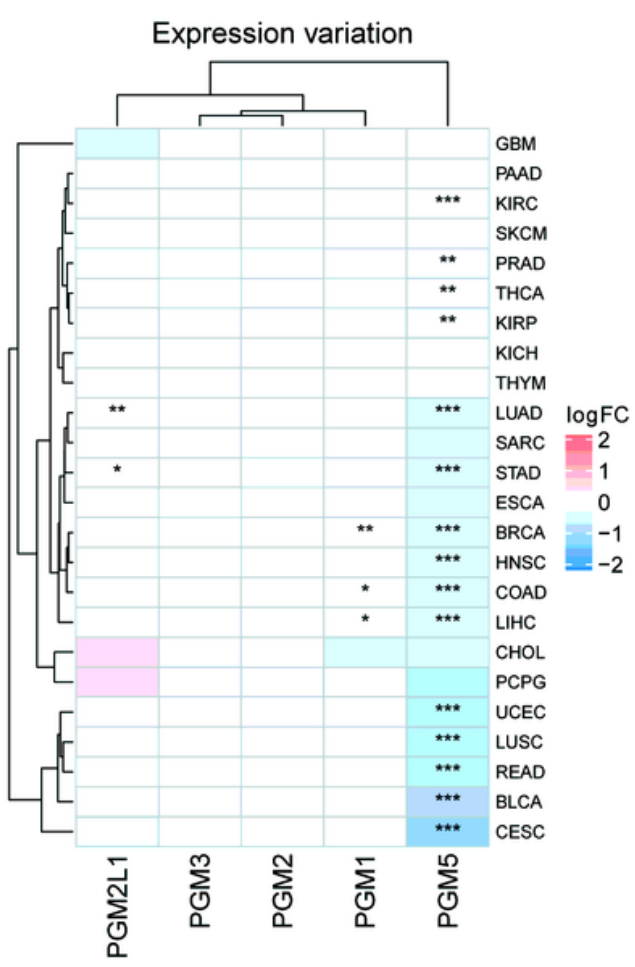

B
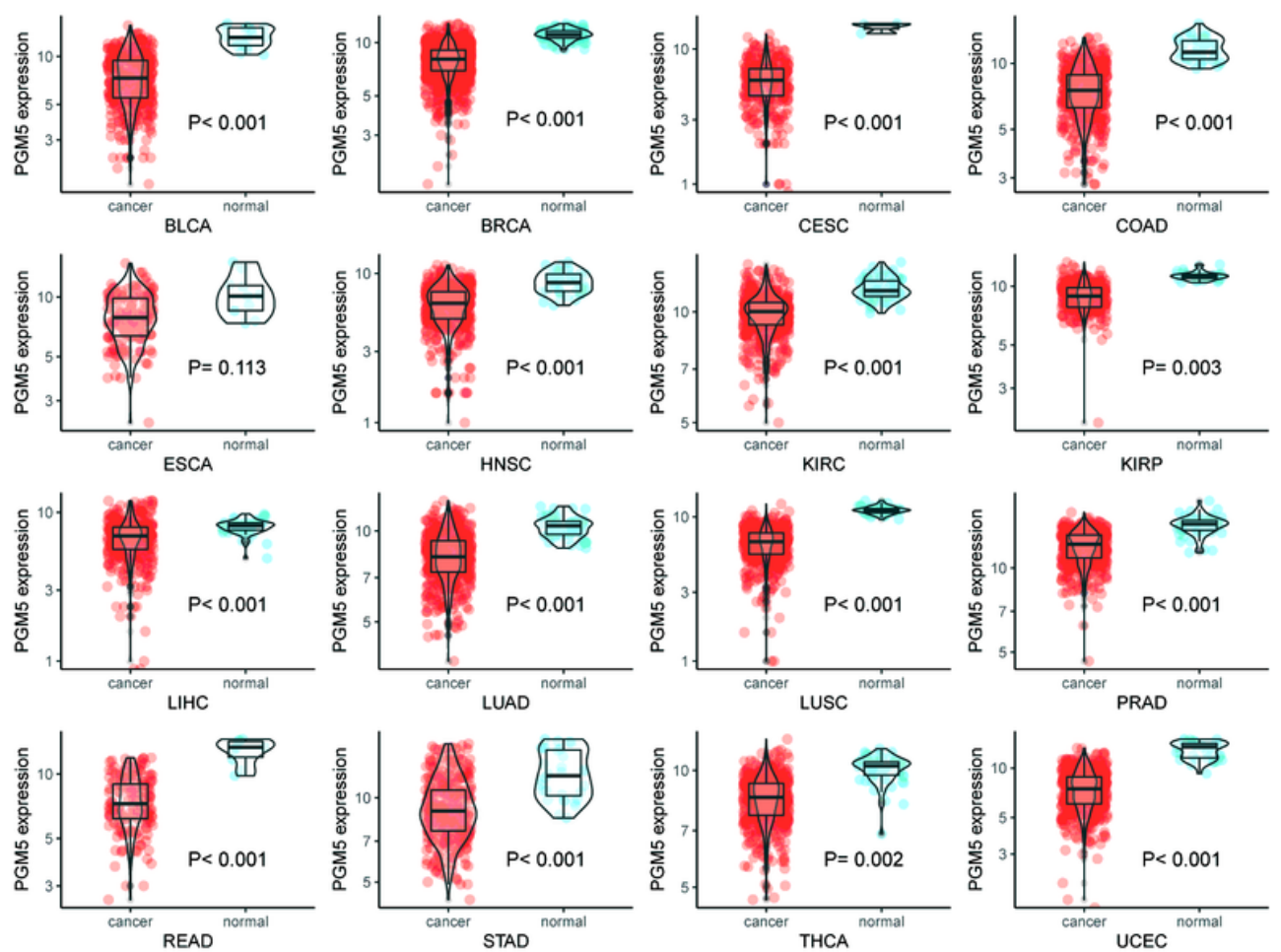

Figure 1

Expression profile of PGMs across different cancer types. (A) Expression of PGMs in different cancer and normal samples. The color in heatmap represents the log2 fold change value between cancer and normal. The blue color represents the low expression in cancer, whereas the red color represents the high expression in cancer. The * sign represents degree of statistical significance, $P<0.05, * \star P<0.01$, *** $P<0.001$. (B) Expression of PGM5 in 16 types of cancers between cancer and normal tissues. 


\begin{tabular}{|r|}
\hline Analysis Type by Cancer \\
\hline Bladder cancer \\
\hline Brain and CNS cancer \\
\hline Breast cancer \\
\hline Cervival cancer \\
\hline Colorectal cancer \\
\hline Esophageal cancer \\
\hline Gastric cancer \\
\hline Head and Neck cancer \\
\hline Kidney cancer \\
\hline Leukemia \\
\hline Liver cancer \\
\hline Lung cancer \\
\hline Lymphoma \\
\hline Melanoma \\
\hline Myeloma \\
\hline Other cancer \\
\hline Ovarian cancer \\
\hline Pancreatic cancer \\
\hline Prostate cancer \\
\hline Sarcoma \\
\hline Total Unique Analyses \\
\hline
\end{tabular}

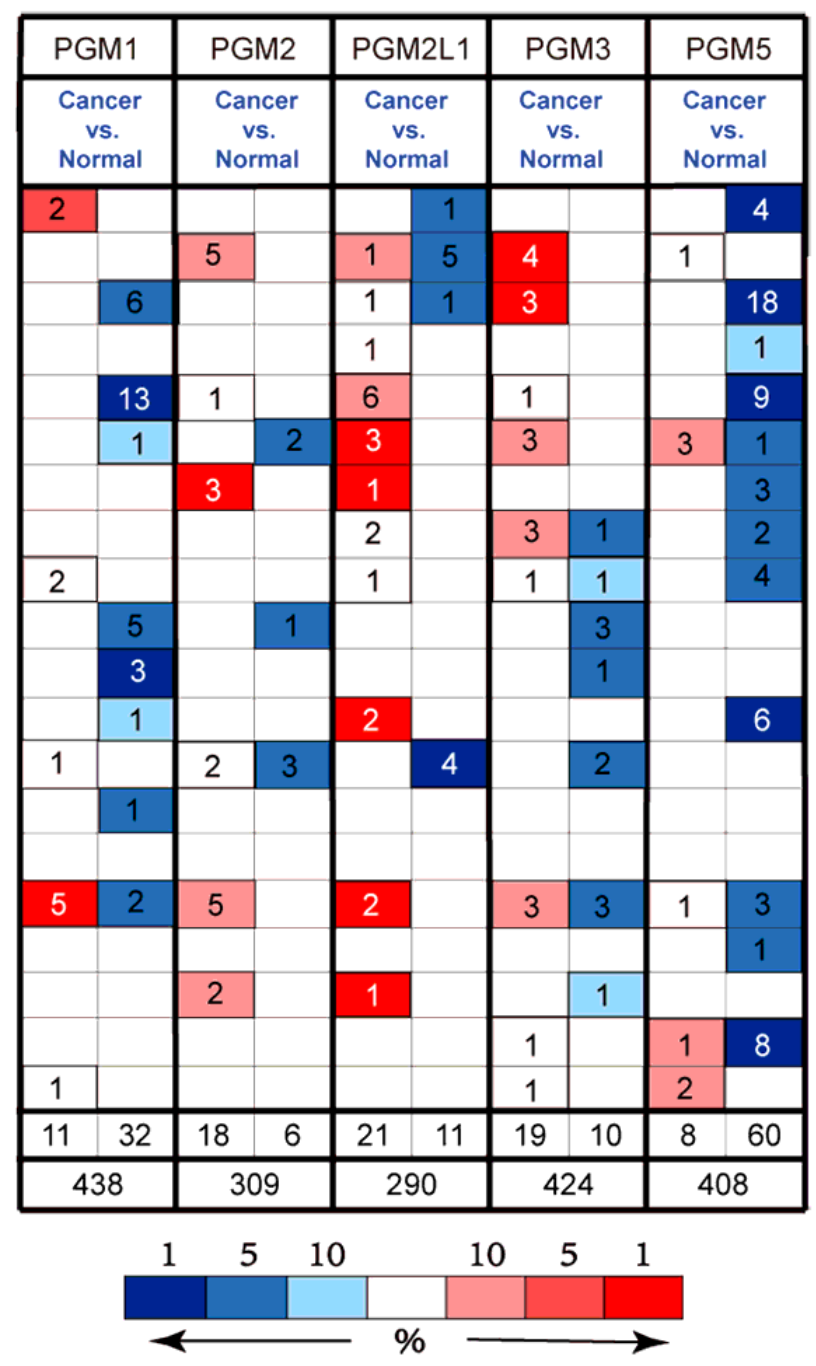

Figure 2

Expression of PGMs in various cancers from Oncomine database. The blue color represents the low expression, whereas the red color represents the high expression.

High

Medium

Low

Not detected

\begin{tabular}{|c|c|c|c|c|c|c|c|c|c|c|c|c|c|c|c|c|c|c|c|c|c|c|c|c|c|c|c|c|c|c|c|c|c|c|c|c|c|}
\hline \multicolumn{3}{|c|}{ LUCA } & \multicolumn{4}{|c|}{ COCA } & \multicolumn{4}{|c|}{ STCA } & \multicolumn{4}{|c|}{ LIHC } & \multicolumn{4}{|c|}{ RACA } & \multicolumn{4}{|c|}{ PRCA } & \multicolumn{3}{|c|}{ BRCA } & \multicolumn{3}{|c|}{ CECA } & \multicolumn{4}{|c|}{ ENCA } & \multicolumn{4}{|c|}{ melanoma } & \multirow[b]{2}{*}{ PGM1 } \\
\hline & 4 & 7 & & & 1 & 10 & 1 & 2 & 3 & 5 & & 6 & 3 & 2 & & 5 & 4 & 3 & & 1 & 5 & 5 & 4 & 7 & & & 4 & 8 & & 1 & 4 & 7 & & 2 & 6 & 4 & \\
\hline & & 12 & & & & 12 & & & 1 & 8 & & 1 & 1 & 8 & 1 & 3 & 4 & 4 & & & & 12 & & & 11 & 1 & 1 & 10 & & 3 & 5 & 4 & 8 & 3 & 1 & & PGM2 \\
\hline 1 & 3 & 5 & & & & 12 & & & 2 & 10 & & & & 12 & & & & 10 & & & & 12 & & 1 & 11 & 1 & 1 & 10 & & & 2 & 10 & & 2 & & 10 & PGM2L1 \\
\hline 3 & 3 & 6 & & 5 & 6 & 1 & & 1 & 2 & 8 & & & 3 & 5 & & 1 & 3 & 8 & 1 & 10 & 1 & & 7 & 3 & 1 & 3 & 5 & 4 & & 6 & 4 & 1 & & 8 & 3 & & PGM3 \\
\hline 1 & 2 & 7 & & & & 11 & & & 2 & 9 & 1 & 3 & 2 & 5 & & 6 & 5 & 1 & & 5 & 5 & 1 & & 3 & 7 & & & 10 & & 1 & 2 & 7 & & 1 & 1 & 10 & PGM5 \\
\hline \multicolumn{3}{|c|}{ glioma } & \multicolumn{4}{|c|}{ THCA } & \multicolumn{4}{|c|}{ HNSC } & \multicolumn{4}{|c|}{ carcinoid } & \multicolumn{4}{|c|}{ PACA } & \multicolumn{4}{|c|}{ URCA } & \multicolumn{3}{|c|}{ TECA } & \multicolumn{3}{|c|}{ OV } & \multicolumn{4}{|c|}{ SKCA } & \multicolumn{4}{|c|}{ lymphoma } & \\
\hline & & & & & & & & & & & & & & & & & & & & & & & & & & & & & & & & & & & & & \\
\hline & 2 & 10 & & 1 & 3 & & & 1 & 2 & 1 & & & 1 & 3 & & & 3 & 8 & & 1 & 2 & 9 & & 5 & 4 & 2 & 3 & 6 & & & 3 & 8 & & & & 12 & PGM1 \\
\hline 1 & 2 & 7 & 3 & 1 & & & & 3 & & 1 & & & 1 & 3 & & & 2 & 9 & & 2 & 2 & 6 & & & 12 & & 2 & 10 & 1 & 1 & & 10 & 1 & 4 & & 7 & PGM2 \\
\hline & 1 & 11 & & & & 4 & & & & 3 & & & & 3 & & 1 & 2 & 9 & & & & 12 & & 1 & 10 & 1 & & 10 & & & & 12 & & & & 12 & PGM2L1 \\
\hline 10 & 1 & & & 2 & 1 & 1 & & 2 & 1 & & & 1 & 3 & & & & 5 & 6 & & 4 & 5 & 3 & 6 & 3 & 2 & 4 & 6 & 2 & & & 4 & 6 & & & 2 & 10 & PGM3 \\
\hline 1 & 1 & 10 & & 1 & & 3 & & & 1 & 3 & & & & 4 & & & 1 & 10 & & & 3 & 9 & & 2 & 9 & 1 & 3 & 8 & & 1 & & 9 & & & & 12 & PGM5 \\
\hline
\end{tabular}

Figure 3 
PGMs protein expression across various cancer types. The expression of each gene in each cancer was divided into four groups of high expression (red color), medium expression (orange color), low expression (yellow color) and not detected (grey color).
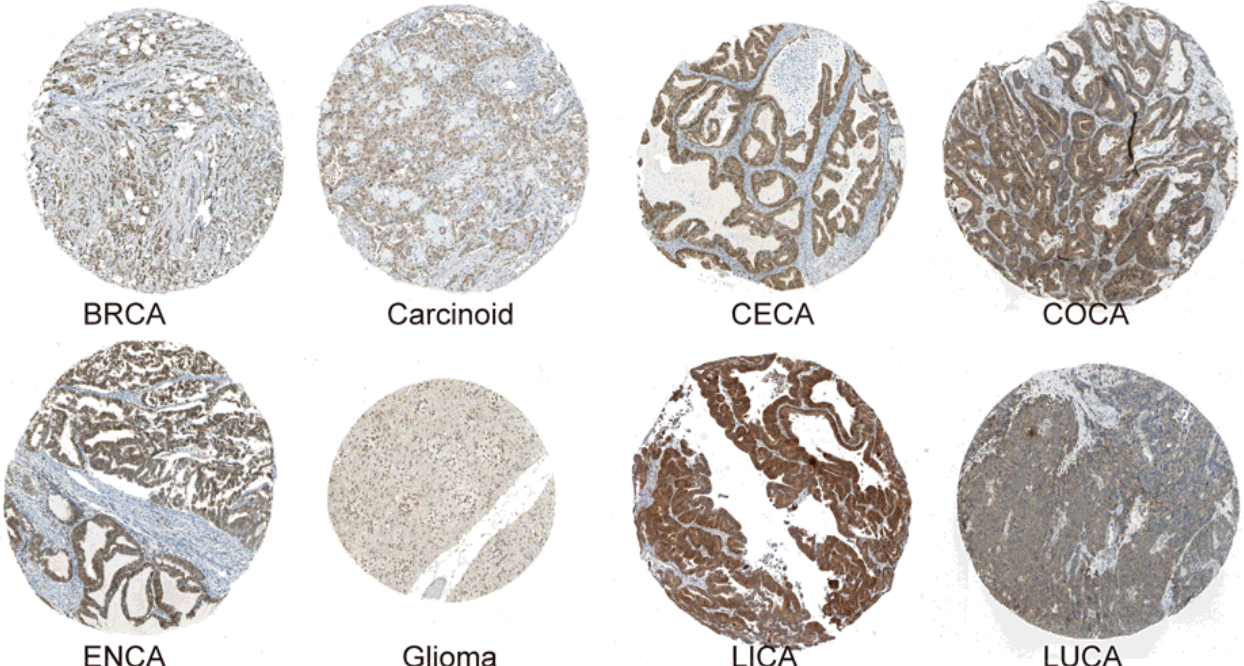

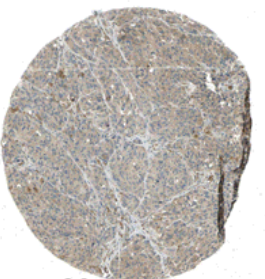

Melanoma

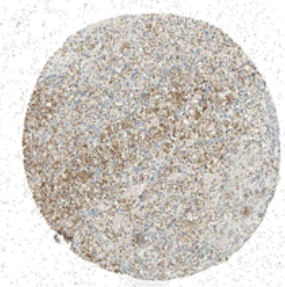

RECA

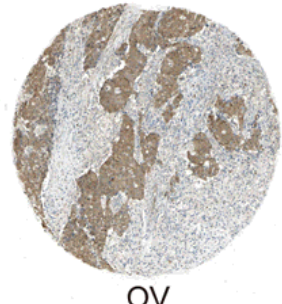

OV

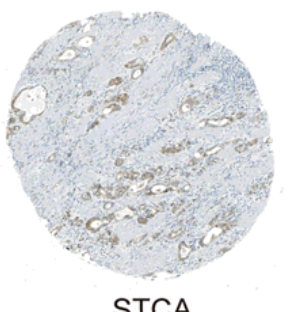

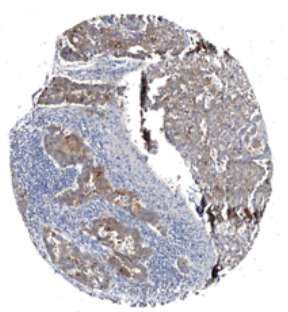

PACA

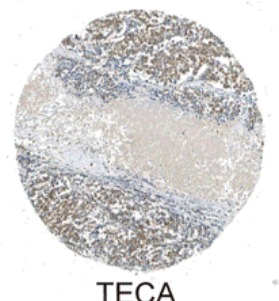

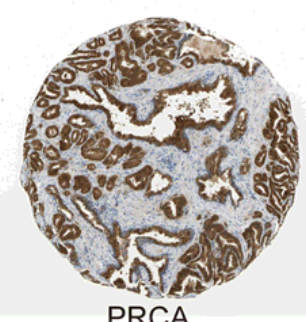

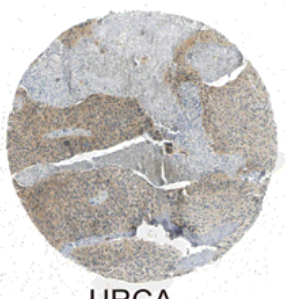

URCA

Figure 4

PGM3 protein expression in 16 cancer types based on immunohischemistry staining results from 'The Human Protein Atlas' database. The 16 cancer types include BRCA (Breast cancer), Carcinoid, CECA (Cervical cancer), COCA (Colorectal cancer), ENCA (Endometrial cancer), Glioma, LICA (Liver cancer), LUCA (Lung cancer), Melanoma, OV (Ovarian cancer), PACA (Pancreatic cancer), PRCA (Prostate cancer), RECA (Renal cancer), STCA (Stomach cancer), TECA (Testis cancer), URCA (Urothelial cancer). 


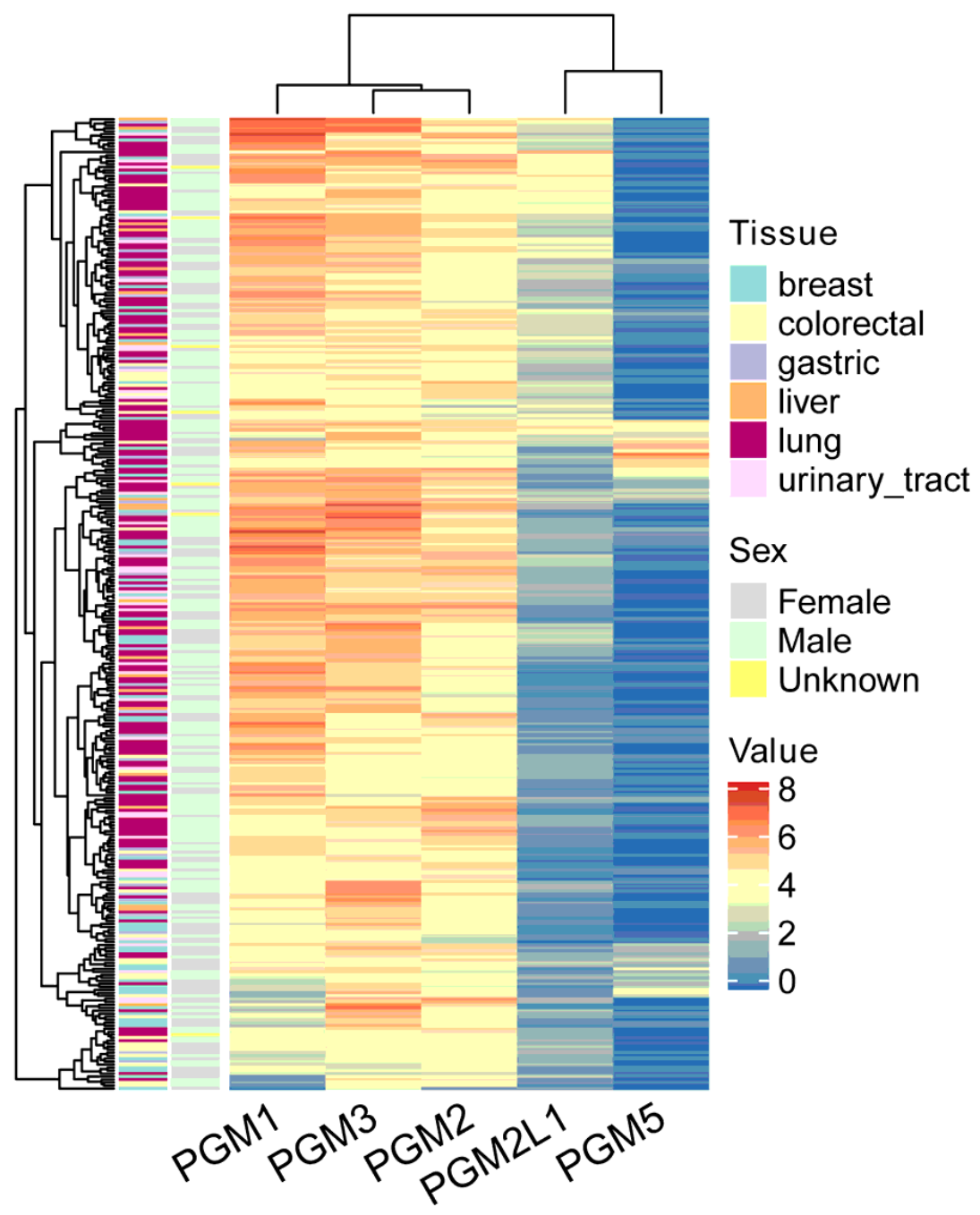

Figure 5

The expression profile of PGMs in 6 cell lines (breast, colorectal, gastric, liver, lung and urinary tract) from CCLE database. The blue color represents the low expression, whereas the red color represents the high expression. 


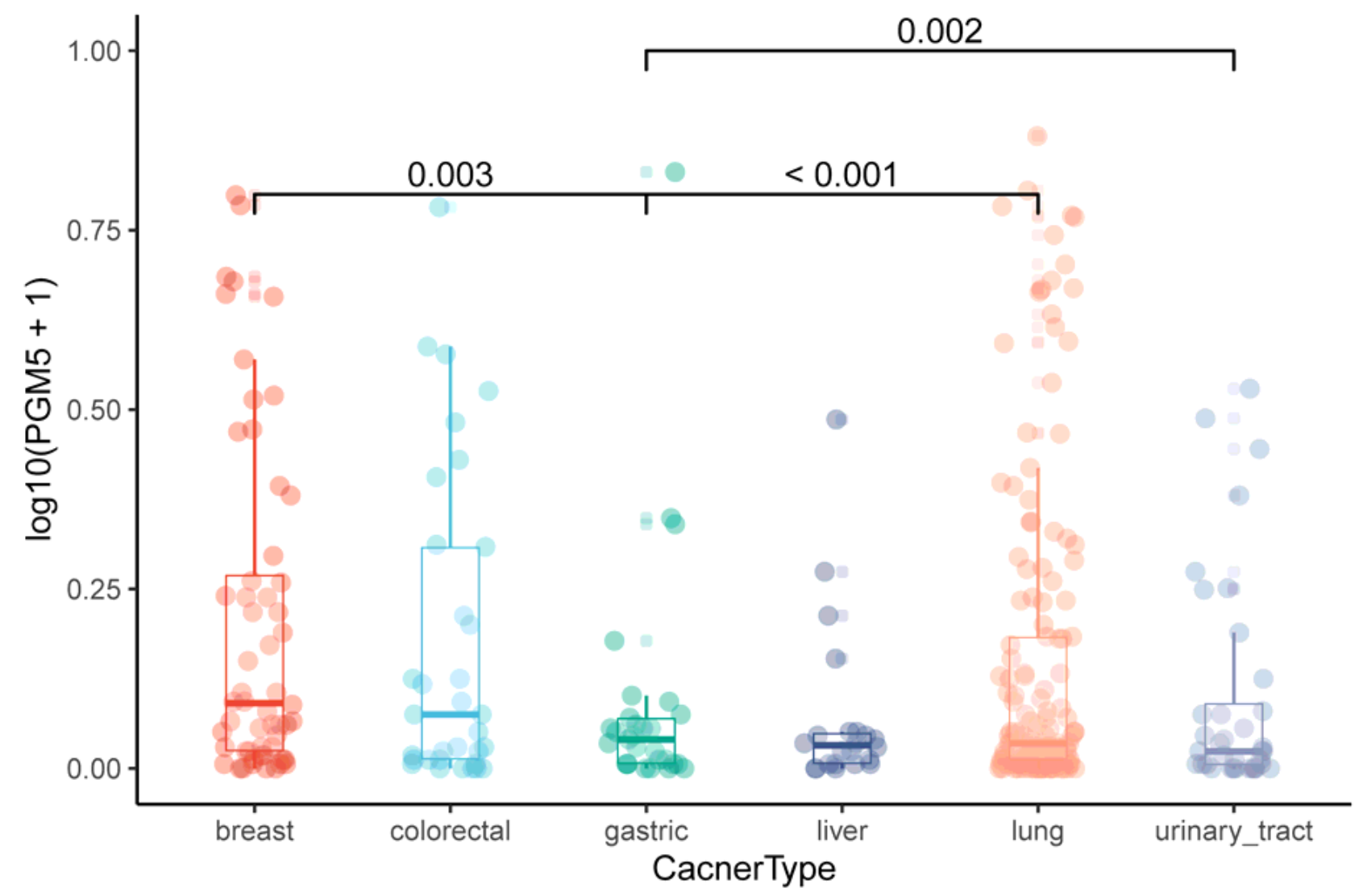

Figure 6

The expression of PGM5 gene in different cell lines (breast, colorectal, gastric, liver, lung and urinary tract) in CCLE database.

A

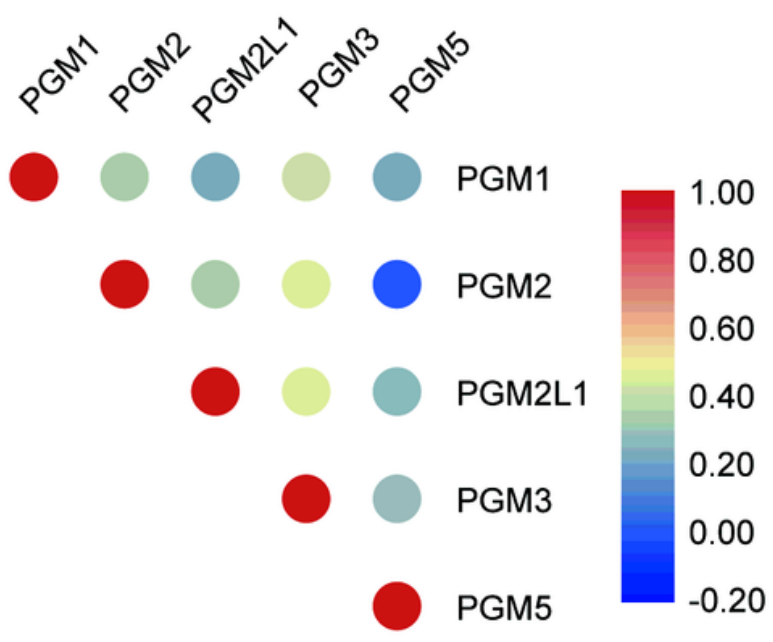

B

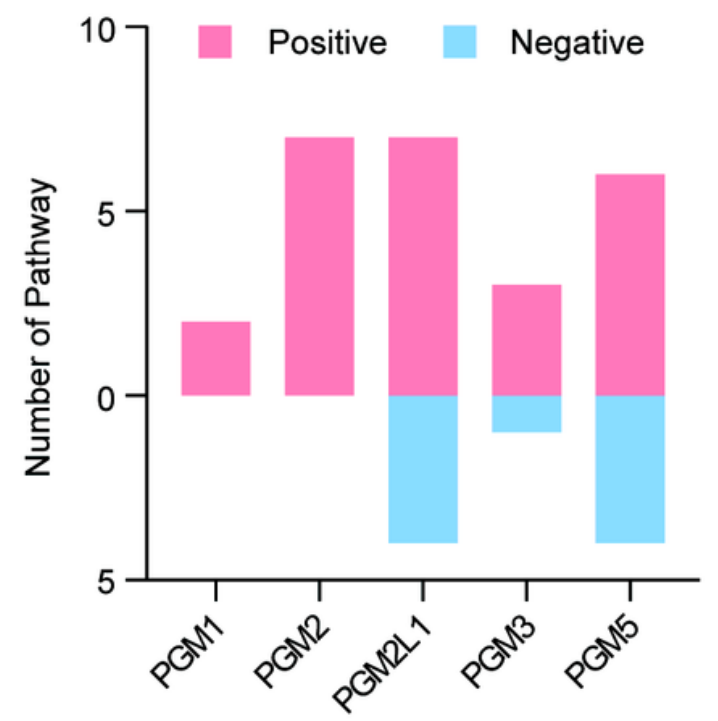

Figure 7

Association of PGMs expression with cancer-related pathways. (A) The number of significantly correlated pathways in each individual PGMs. (B) Correlation between the expression of different PGMs. 
A Cancer: TGCT

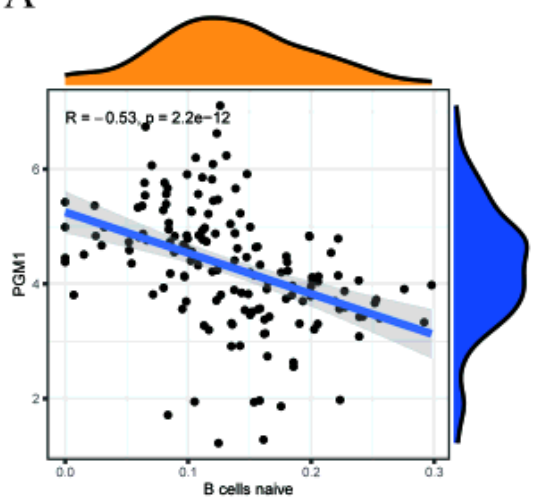

D Cancer: THYM

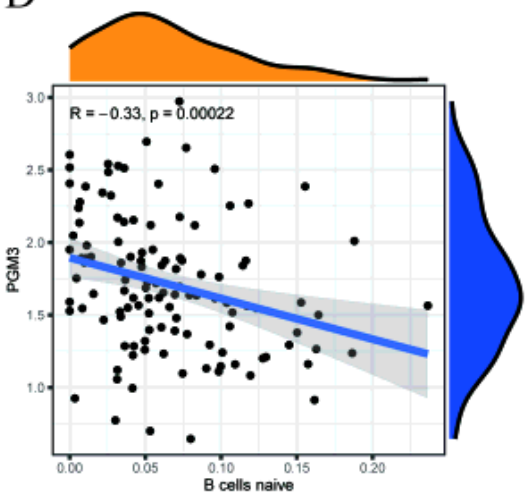

B Cancer: TGCT

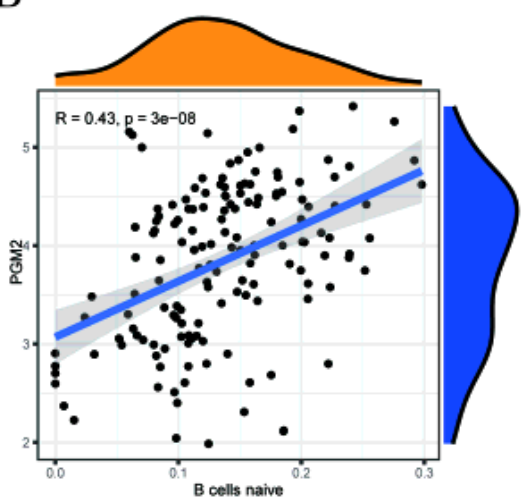

E Cancer: UCS

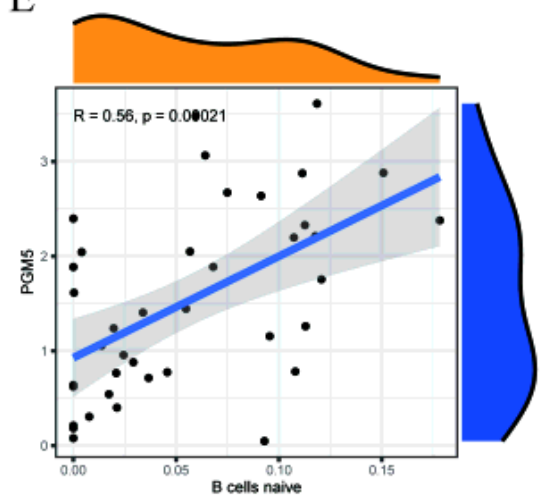

$C$ Cancer: PRAD

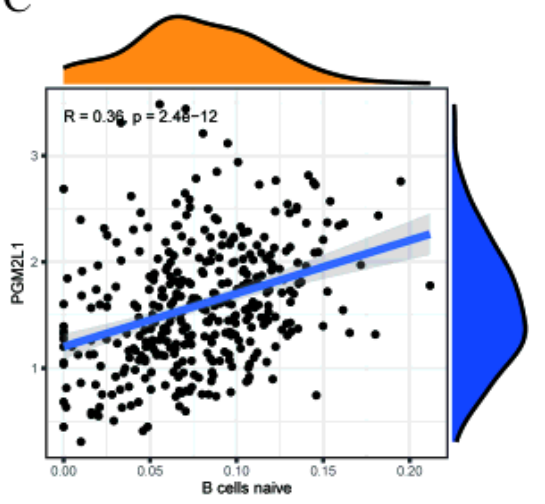

Figure 8

Correlation between PGMs expression and naïve B cell infiltration level in different cancers from TIMER. (A) Correlation between PGM1 expression and naïve B cell infiltration level in TGCT. (B) Correlation between PGM2 expression and naïve B cell infiltration level in TGCT. (C) Correlation between PGM2L1 expression and naïve B cell infiltration level in PRAD. (D) Correlation between PGM3 expression and naïve B cell infiltration level in THYM. (E) Correlation between PGM5 expression and naïve B cell infiltration level in UCS. 


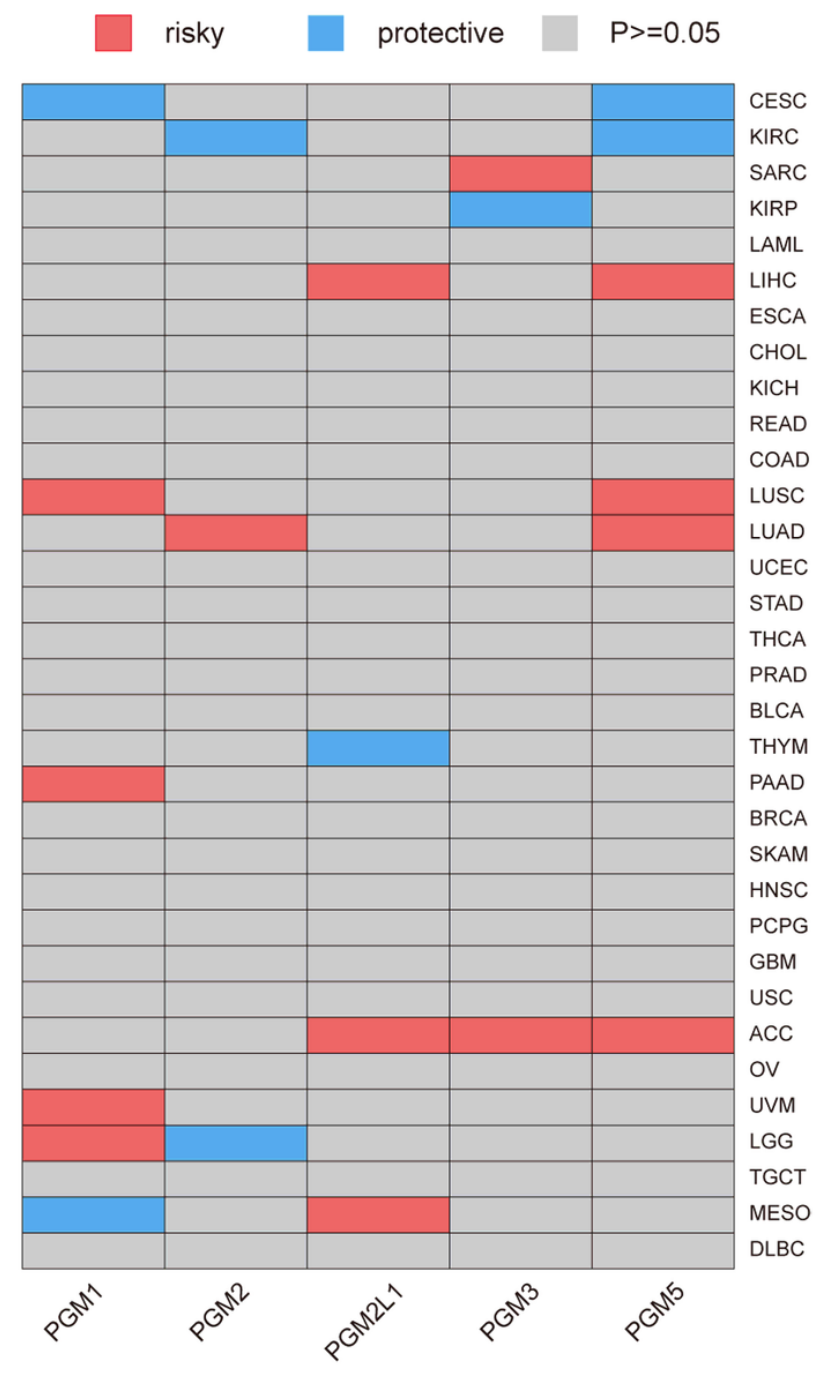

\section{Figure 9}

Correlation between PGMs expression and survival of different cancers. Red color represents high risk of death whereas blue color represents low risk of death. 


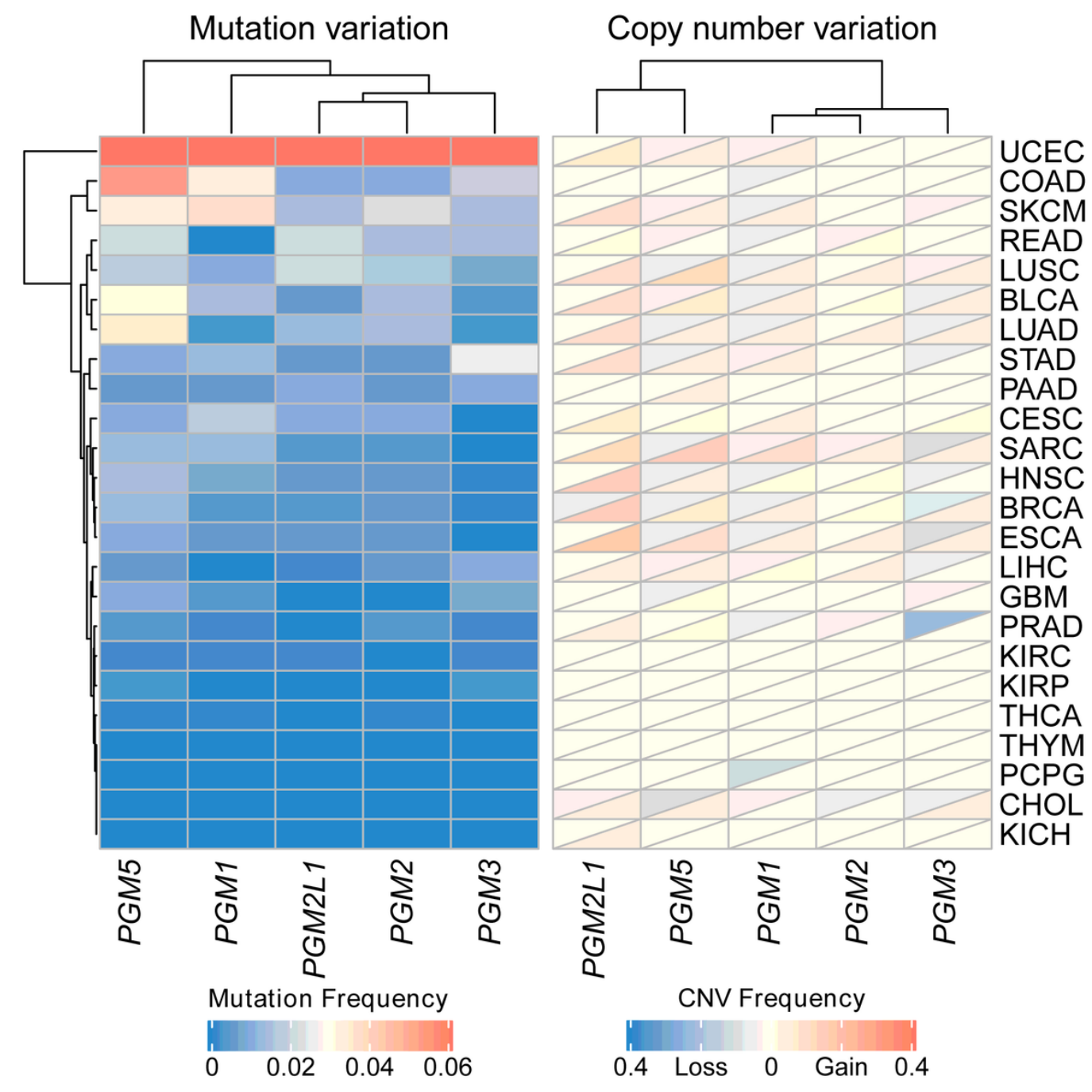

Figure 10

Pan-cancer genetic alternations of PGMs. (A) Mutation frequency of PGMs in different cancers. Red color represents high mutation frequency whereas blue color represents low mutation frequency. (B) The copy number variations frequency of PGMs in different cancers. The upper part of each grid shows the deletion frequency, and the bottom part shows the amplification frequency. 


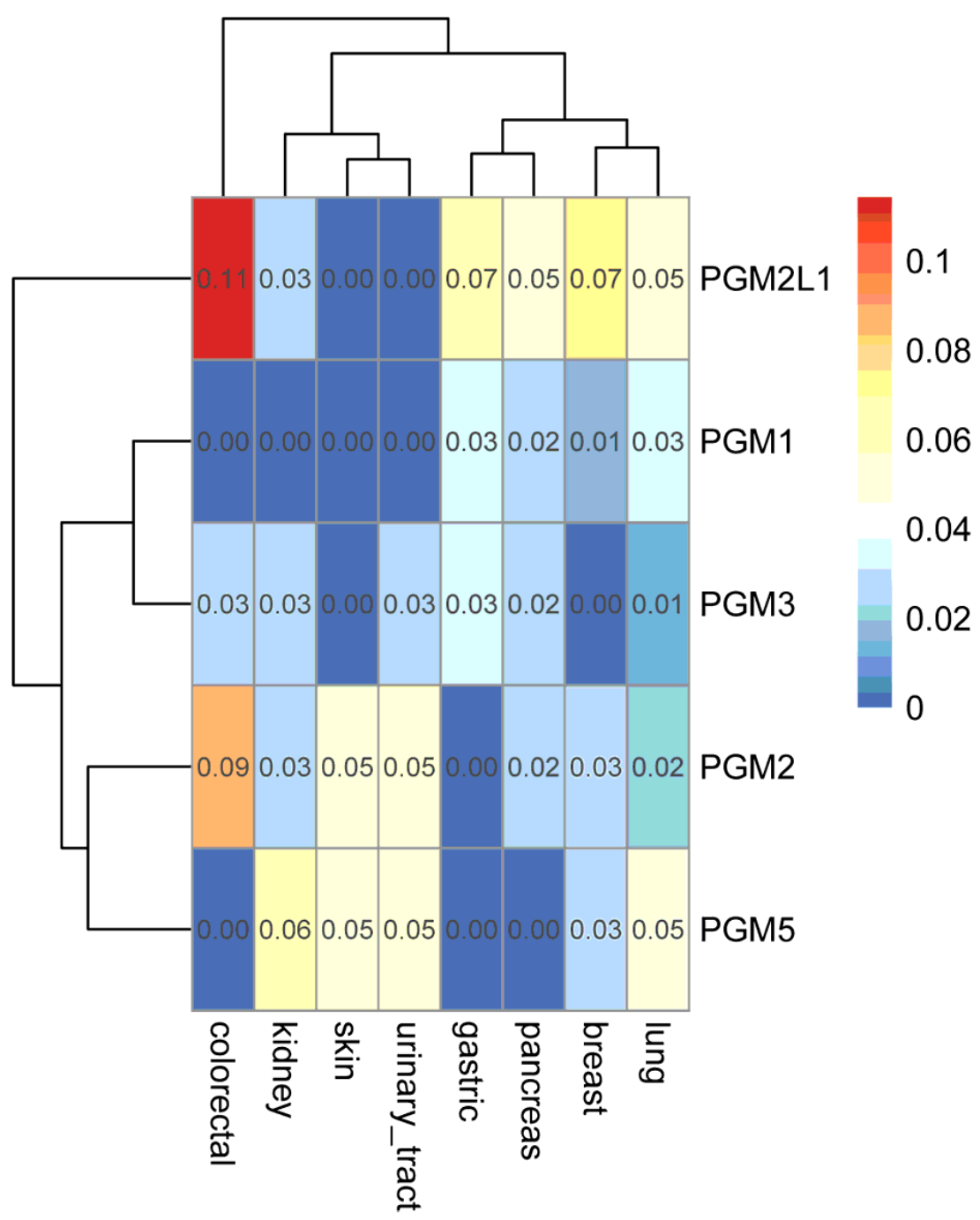

Figure 11

Mutation frequency of PGMs in different cancer cell lines (colorectal, kidney, skin, urinary tract, gastric, pancreas, breast and lung) from CCLE database. Red color represents high mutation frequency whereas blue color represents low mutation frequency. 


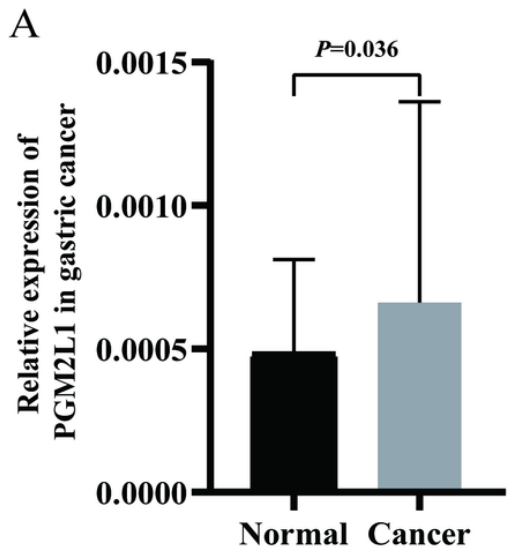

$\mathrm{C}$

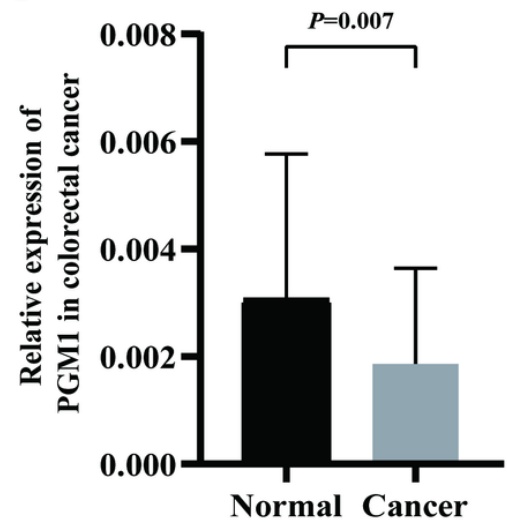

B

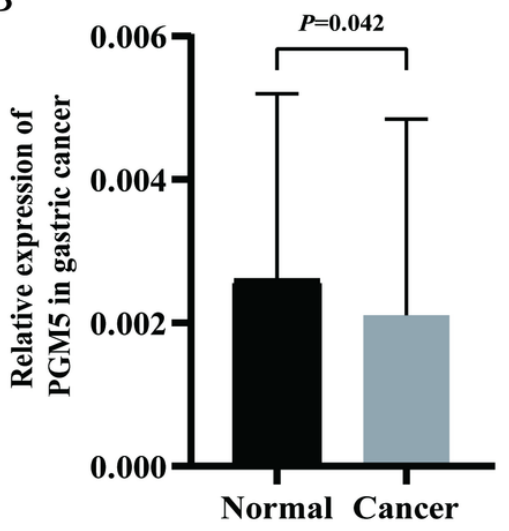

$\mathrm{D}$

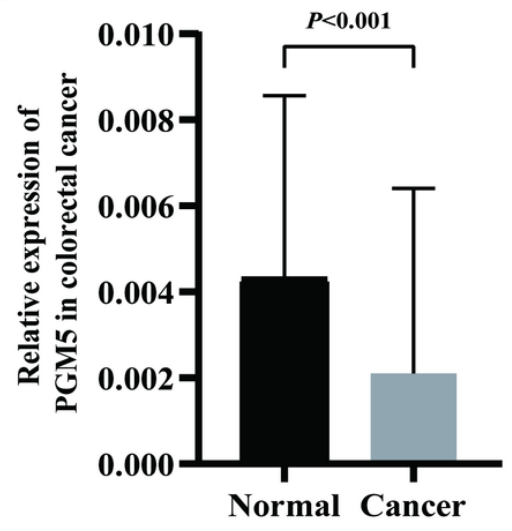

Figure 12

Validation of the TCGA and Oncomine analysis which indicated that the expression of PGM2L1 in gastric cancer was upregulated, the expression of PGM5 in gastric cancer was downregulated, and the expression of PGM1 and PGM5 in colorectal cancer were both downregulated using the qRT-PCR experiments. $P<0.05$ was considered statistically significant. (A) The relative expression of PGM2L1 in gastric cancer. (B) The relative expression of PGM5 in gastric cancer. (C) The relative expression of PGM1 in colorectal cancer. (D) The relative expression of PGM5 in colorectal cancer.

\section{Supplementary Files}

This is a list of supplementary files associated with this preprint. Click to download.

- Additionalfile.docx 